IJMMS 29:4 (2002) 217-238

PII. S016117120200501X

http://ijmms.hindawi.com

(c) Hindawi Publishing Corp.

\title{
MATRIX METHOD FOR SOLVING LINEAR COMPLEX VECTOR FUNCTIONAL EQUATIONS
}

\author{
ICE B. RISTESKI
}

Received 24 April 2000

\begin{abstract}
We give a new matrix method for solving both homogeneous and nonhomogeneous linear complex vector functional equations with constant complex coefficients.
\end{abstract}

2000 Mathematics Subject Classification: 39B32, 39B52.

1. Introduction and preliminaries. In the previous investigation [1] the solution of one class of homogeneous complex vector functional equations is obtained by the method of intersections, while in [2] several classes of homogeneous complex vector functional equations are solved on the basis of the well-known method of elimination of variables. For the purpose of expanding our investigations, here we will propose a matrix method suitable for both homogeneous and nonhomogeneous complex vector functional equations with constant complex coefficients.

Now we will introduce the following notation. Let $\mathscr{V}$ be a finite-dimensional complex vector space and let a mapping $f: \mathscr{V}^{n} \mapsto \mathscr{V}$ exists. Throughout, $\mathbf{Z}_{i}(1 \leq i \leq n)$ are vectors in $\mathscr{V}$. We assume that $\mathbf{Z}_{i}=\left(z_{i 1}(t), \ldots, z_{i n}(t)\right)^{T}$, where $z_{i j}(t)(1 \leq i \leq n)$ are complex functions and $\mathbf{O}=(0,0, \ldots, 0)^{T}$ is the zero vector in $\mathscr{V}$.

Let $A$ be an $n \times n$ matrix. Suppose that by elementary transformations the matrix $A$ is transformed into $A=P_{1} D P_{2}$, where $P_{1}$ and $P_{2}$ are regular matrices and $D$ is a diagonal matrix with diagonal entries 0 and 1 such that the number of units is equal to the rank of the matrix $A$. The matrix $B=P_{2}^{-1} D P_{1}^{-1}$ satisfies the equality $A B A=A$. This means that the matrix equation $A X A=A$ has at least one solution for $X$.

If $A$ satisfies the identity

$$
A^{r}+k_{1} A^{r-1}+\cdots+k_{r-1} A=O \quad\left(k_{r-1} \neq 0\right),
$$

where $O$ is the $n \times n$ zero matrix, then the matrix

$$
X=-\frac{1}{k_{r-1}}\left(A^{r-2}+k_{1} A^{r-3}+\cdots+k_{r-2} I\right),
$$

where $I$ is the unit $n \times n$ matrix, is also a solution of the equation $A X A=A$.

Now we will prove the following theorem.

THEOREM 1.1. If $B$ satisfies the condition $A B A=A$, then

(1) $A X=O \Leftrightarrow X=(I-B A) Q,(X$ and $Q$ are $n \times m$ matrices $)$;

(2) $X A=O \Leftrightarrow X=Q(I-A B)$, ( $X$ and $Q$ are $m \times n$ matrices ;

(3) $A X A=A \Leftrightarrow X=B+Q-B A Q A B$ ( $X$ and $Q$ are $n \times n$ matrices); 
(4) $A X=A \Leftrightarrow X=I+(I-B A) Q$;

(5) $X A=A \Leftrightarrow X=I+Q(I-A B)$.

Proof. The theorem will be proved only for the case (3), because the other cases can be proved analogously.

(3) Let $X=B+Q-B A Q A B$. Further it holds that

$$
\begin{gathered}
A X A=A B A+A Q A-A B A Q A B A, \quad A B A=A \\
\Longrightarrow A X A=A+A Q A-A Q A \Longrightarrow A X A=A .
\end{gathered}
$$

Conversely, assume that $A X A=A$, then it holds that

$$
B+(X-B)-B A(X-B) A B=X-B A X A B+B A B A B=X-B A B+B A B=X .
$$

Thus

$$
A X A=A \Longrightarrow X=B+Q-B A Q A B, \text { for } Q=X-B .
$$

If $X$ is an $n \times k$ matrix, according to Theorem 1.1(1) all the solutions of the homogeneous system of equations

$$
A\left[\begin{array}{c}
x_{1} \\
x_{2} \\
\vdots \\
x_{n}
\end{array}\right]=O
$$

have the following form

$$
\left[\begin{array}{c}
x_{1} \\
x_{2} \\
\vdots \\
x_{n}
\end{array}\right]=(I-B A)\left[\begin{array}{c}
u_{1} \\
u_{2} \\
\vdots \\
u_{n}
\end{array}\right]
$$

where $u_{1}, \ldots, u_{n}$ are arbitrary.

2. Solution of homogeneous functional equations. Now we will prove the following results.

THEOREM 2.1. The general solution of the basic cyclic complex vector functional equation with complex constant coefficients

$$
E(f) \equiv \sum_{i=1}^{n} a_{i} f\left(\mathbf{Z}_{i}, \mathbf{Z}_{i+1}, \ldots, \mathbf{Z}_{i+n-1}\right)=\mathbf{O} \quad\left(\mathbf{Z}_{n+i} \equiv \mathbf{Z}_{i}\right),
$$

where $a_{i}(1 \leq i \leq n)$ are complex constants, is given by the following formula:

$$
\left[\begin{array}{c}
f\left(\mathbf{Z}_{1}, \mathbf{Z}_{2}, \ldots, \mathbf{Z}_{n}\right) \\
f\left(\mathbf{Z}_{2}, \mathbf{Z}_{3}, \ldots, \mathbf{Z}_{1}\right) \\
\vdots \\
f\left(\mathbf{Z}_{n}, \mathbf{Z}_{1}, \ldots, \mathbf{Z}_{n-1}\right)
\end{array}\right]=B\left[\begin{array}{c}
h\left(\mathbf{Z}_{1}, \mathbf{Z}_{2}, \ldots, \mathbf{Z}_{n}\right) \\
h\left(\mathbf{Z}_{2}, \mathbf{Z}_{3}, \ldots, \mathbf{Z}_{1}\right) \\
\vdots \\
h\left(\mathbf{Z}_{n}, \mathbf{Z}_{1}, \ldots, \mathbf{Z}_{n-1}\right)
\end{array}\right],
$$


where

$$
A=\left[\begin{array}{cccc}
a_{1} & a_{2} & \cdots & a_{n} \\
a_{n} & a_{1} & \cdots & a_{n-1} \\
\vdots & & & \\
a_{2} & a_{3} & \cdots & a_{1}
\end{array}\right], \quad B=\left[\begin{array}{cccc}
b_{1} & b_{2} & \cdots & b_{n} \\
b_{n} & b_{1} & \cdots & b_{n-1} \\
\vdots & & & \\
b_{2} & b_{3} & \cdots & b_{1}
\end{array}\right]
$$

are nonzero $n \times n$ cyclic matrices with complex constant elements such that

$$
A B=O
$$

where $O$ is the $n \times n$ zero matrix and $h$ is an arbitrary complex vector function with values in $\mathscr{V}$.

Proof. If we permute successively the vectors in (2.1), we get

$$
\begin{aligned}
& a_{1} f\left(\mathbf{Z}_{1}, \mathbf{Z}_{2}, \ldots, \mathbf{Z}_{n}\right)+a_{2} f\left(\mathbf{Z}_{2}, \mathbf{Z}_{3}, \ldots, \mathbf{Z}_{n}, \mathbf{Z}_{1}\right)+\cdots+a_{n} f\left(\mathbf{Z}_{n}, \mathbf{Z}_{1}, \ldots, \mathbf{Z}_{n-1}\right)=\mathbf{O}, \\
& a_{n} f\left(\mathbf{Z}_{1}, \mathbf{Z}_{2}, \ldots, \mathbf{Z}_{n}\right)+a_{1} f\left(\mathbf{Z}_{2}, \mathbf{Z}_{3}, \ldots, \mathbf{Z}_{n}, \mathbf{Z}_{1}\right)+\cdots+a_{n-1} f\left(\mathbf{Z}_{n}, \mathbf{Z}_{1}, \ldots, \mathbf{Z}_{n-1}\right)=\mathbf{O}, \\
& \vdots \\
& a_{2} f\left(\mathbf{Z}_{1}, \mathbf{Z}_{2}, \ldots, \mathbf{Z}_{n}\right)+a_{3} f\left(\mathbf{Z}_{2}, \mathbf{Z}_{3}, \ldots, \mathbf{Z}_{n}, \mathbf{Z}_{1}\right)+\cdots+a_{1} f\left(\mathbf{Z}_{n}, \mathbf{Z}_{1}, \ldots, \mathbf{Z}_{n-1}\right)=\mathbf{O},
\end{aligned}
$$

that is, in a matrix form

$$
A F=0
$$

where $A$ is given by (2.3),

$$
F=\left[\begin{array}{c}
f\left(\mathbf{Z}_{1}, \mathbf{Z}_{2}, \ldots, \mathbf{Z}_{n}\right) \\
f\left(\mathbf{Z}_{2}, \mathbf{Z}_{3}, \ldots, \mathbf{Z}_{1}\right) \\
\vdots \\
f\left(\mathbf{Z}_{n}, \mathbf{Z}_{1}, \ldots, \mathbf{Z}_{n-1}\right)
\end{array}\right], \quad \mathcal{O}=\left[\begin{array}{c}
\mathbf{O} \\
\mathbf{O} \\
\vdots \\
\mathbf{O}
\end{array}\right] .
$$

We will write $f \in F$ to express the first equality of (2.7).

The necessary and sufficient condition for system (2.6) to have a nontrivial solution is

$$
\operatorname{det} A=0
$$

Let

$$
f\left(\mathbf{Z}_{1}, \mathbf{Z}_{2}, \ldots, \mathbf{Z}_{n}\right)=\sum_{i=1}^{n} b_{i} h\left(\mathbf{Z}_{i}, \mathbf{Z}_{i+1}, \ldots, \mathbf{Z}_{i+n-1}\right) \quad\left(\mathbf{Z}_{n+i} \equiv \mathbf{Z}_{i}\right),
$$

where $b_{i}(1 \leq i \leq n)$ are complex constants such that the matrix $B$, defined by (2.3), satisfies (2.4) and $h$ is an arbitrary complex vector function with values in $\mathscr{V}$. 
By a cyclic permutation of the vectors in (2.9), we obtain

$$
\begin{aligned}
& f\left(\mathbf{Z}_{1}, \mathbf{Z}_{2}, \ldots, \mathbf{Z}_{n}\right)=b_{1} h\left(\mathbf{Z}_{1}, \mathbf{Z}_{2}, \ldots, \mathbf{Z}_{n}\right)+b_{2} h\left(\mathbf{Z}_{2}, \mathbf{Z}_{3}, \ldots, \mathbf{Z}_{1}\right)+\cdots+b_{n} h\left(\mathbf{Z}_{n}, \mathbf{Z}_{1}, \ldots, \mathbf{Z}_{n-1}\right), \\
& f\left(\mathbf{Z}_{2}, \mathbf{Z}_{3}, \ldots, \mathbf{Z}_{1}\right)=b_{n} h\left(\mathbf{Z}_{1}, \mathbf{Z}_{2}, \ldots, \mathbf{Z}_{n}\right)+b_{1} h\left(\mathbf{Z}_{2}, \mathbf{Z}_{3}, \ldots, \mathbf{Z}_{1}\right)+\cdots+b_{n-1} h\left(\mathbf{Z}_{n}, \mathbf{Z}_{1}, \ldots, \mathbf{Z}_{n-1}\right), \\
& \vdots \\
& f\left(\mathbf{Z}_{n}, \mathbf{Z}_{1}, \ldots, \mathbf{Z}_{n-1}\right)=b_{2} h\left(\mathbf{Z}_{1}, \mathbf{Z}_{2}, \ldots, \mathbf{Z}_{n}\right)+b_{3} h\left(\mathbf{Z}_{2}, \mathbf{Z}_{3}, \ldots, \mathbf{Z}_{1}\right)+\cdots+b_{1} h\left(\mathbf{Z}_{n}, \mathbf{Z}_{1}, \ldots, \mathbf{Z}_{n-1}\right),
\end{aligned}
$$

that is, in a matrix form

$$
F=B H,
$$

where

$$
H=\left[\begin{array}{c}
h\left(\mathbf{Z}_{1}, \mathbf{Z}_{2}, \ldots, \mathbf{Z}_{n}\right) \\
h\left(\mathbf{Z}_{2}, \mathbf{Z}_{3}, \ldots, \mathbf{Z}_{1}\right) \\
\vdots \\
h\left(\mathbf{Z}_{n}, \mathbf{Z}_{1}, \ldots, \mathbf{Z}_{n-1}\right)
\end{array}\right]
$$

and $F$ is defined in (2.7).

After a multiplication of (2.11) by $A$, we have

$$
A F=A B H=O H=O,
$$

which means that the function $f \in F$ satisfies the functional equation (2.1) for any $h \in H$.

On the other hand, according to Theorem 1.1(1) each solution of (2.6) has the form

$$
F=(I-\tilde{B} A) H
$$

where $\tilde{B}$ satisfies $A \tilde{B} A=A$. If we put $B=I-\tilde{B} A$, we have $A B=A-A \tilde{B} A=O$ and $B \neq O$ since $\operatorname{det} A=0$. Thus (2.11) where $B$ satisfies (2.4) is the general solution of system (2.6), that is, (2.9) is the general solution of the functional equation (2.1).

EXAMPLE 2.2. By a cyclic permutation of the variables in the functional equation

$$
f\left(\mathbf{Z}_{1}, \mathbf{Z}_{2}, \mathbf{Z}_{3}, \mathbf{Z}_{4}\right)-f\left(\mathbf{Z}_{2}, \mathbf{Z}_{3}, \mathbf{Z}_{4}, \mathbf{Z}_{1}\right)+f\left(\mathbf{Z}_{3}, \mathbf{Z}_{4}, \mathbf{Z}_{1}, \mathbf{Z}_{2}\right)-f\left(\mathbf{Z}_{4}, \mathbf{Z}_{1}, \mathbf{Z}_{2}, \mathbf{Z}_{3}\right)=\mathbf{O},
$$

we obtain the following system:

$$
\begin{array}{r}
f\left(\mathbf{Z}_{1}, \mathbf{Z}_{2}, \mathbf{Z}_{3}, \mathbf{Z}_{4}\right)-f\left(\mathbf{Z}_{2}, \mathbf{Z}_{3}, \mathbf{Z}_{4}, \mathbf{Z}_{1}\right)+f\left(\mathbf{Z}_{3}, \mathbf{Z}_{4}, \mathbf{Z}_{1}, \mathbf{Z}_{2}\right)-f\left(\mathbf{Z}_{4}, \mathbf{Z}_{1}, \mathbf{Z}_{2}, \mathbf{Z}_{3}\right)=\mathbf{O}, \\
-f\left(\mathbf{Z}_{1}, \mathbf{Z}_{2}, \mathbf{Z}_{3}, \mathbf{Z}_{4}\right)+f\left(\mathbf{Z}_{2}, \mathbf{Z}_{3}, \mathbf{Z}_{4}, \mathbf{Z}_{1}\right)-f\left(\mathbf{Z}_{3}, \mathbf{Z}_{4}, \mathbf{Z}_{1}, \mathbf{Z}_{2}\right)+f\left(\mathbf{Z}_{4}, \mathbf{Z}_{1}, \mathbf{Z}_{2}, \mathbf{Z}_{3}\right)=\mathbf{O}, \\
f\left(\mathbf{Z}_{1}, \mathbf{Z}_{2}, \mathbf{Z}_{3}, \mathbf{Z}_{4}\right)-f\left(\mathbf{Z}_{2}, \mathbf{Z}_{3}, \mathbf{Z}_{4}, \mathbf{Z}_{1}\right)+f\left(\mathbf{Z}_{3}, \mathbf{Z}_{4}, \mathbf{Z}_{1}, \mathbf{Z}_{2}\right)-f\left(\mathbf{Z}_{4}, \mathbf{Z}_{1}, \mathbf{Z}_{2}, \mathbf{Z}_{3}\right)=\mathbf{O}, \\
-f\left(\mathbf{Z}_{1}, \mathbf{Z}_{2}, \mathbf{Z}_{3}, \mathbf{Z}_{4}\right)+f\left(\mathbf{Z}_{2}, \mathbf{Z}_{3}, \mathbf{Z}_{4}, \mathbf{Z}_{1}\right)-f\left(\mathbf{Z}_{3}, \mathbf{Z}_{4}, \mathbf{Z}_{1}, \mathbf{Z}_{2}\right)+f\left(\mathbf{Z}_{4}, \mathbf{Z}_{1}, \mathbf{Z}_{2}, \mathbf{Z}_{3}\right)=\mathbf{O} .
\end{array}
$$


The matrix of coefficients of this system is

$$
A=\left[\begin{array}{rrrr}
1 & -1 & 1 & -1 \\
-1 & 1 & -1 & 1 \\
1 & -1 & 1 & -1 \\
-1 & 1 & -1 & 1
\end{array}\right]
$$

Since $\operatorname{det} A=0$, system (2.16) has a nontrivial solution.

For the matrix $A$ there exists a nonzero $4 \times 4$ cyclic matrix $B$, such that $A B=O$, that is,

$$
B=\left[\begin{array}{cccc}
b_{1} & b_{2} & b_{3} & b_{1}-b_{2}+b_{3} \\
b_{1}-b_{2}+b_{3} & b_{1} & b_{2} & b_{3} \\
b_{3} & b_{1}-b_{2}+b_{3} & b_{1} & b_{2} \\
b_{2} & b_{3} & b_{1}-b_{2}+b_{3} & b_{1}
\end{array}\right] .
$$

Therefore the general solution of the given functional equation is

$$
\begin{aligned}
f\left(\mathbf{Z}_{1}, \mathbf{Z}_{2}, \mathbf{Z}_{3}, \mathbf{Z}_{4}\right)= & b_{1} h\left(\mathbf{Z}_{1}, \mathbf{Z}_{2}, \mathbf{Z}_{3}, \mathbf{Z}_{4}\right)+b_{2} h\left(\mathbf{Z}_{2}, \mathbf{Z}_{3}, \mathbf{Z}_{4}, \mathbf{Z}_{1}\right) \\
& +b_{3} h\left(\mathbf{Z}_{3}, \mathbf{Z}_{4}, \mathbf{Z}_{1}, \mathbf{Z}_{2}\right)+\left(b_{1}-b_{2}+b_{3}\right) h\left(\mathbf{Z}_{4}, \mathbf{Z}_{1}, \mathbf{Z}_{2}, \mathbf{Z}_{3}\right),
\end{aligned}
$$

where $h$ is an arbitrary complex vector function with values in $\mathscr{V}$.

THEOREM 2.3. If the matrix A satisfies the condition

$$
A^{m}+\lambda_{1} A^{m-1}+\cdots+\lambda_{m-1} A=O \quad\left(\lambda_{m-1} \neq 0\right),
$$

then the general solution of the functional equation (2.1) is given by

$$
F=\frac{1}{\lambda_{m-1}}\left(A^{m-1}+\lambda_{1} A^{m-2}+\cdots+\lambda_{m-1} I\right) H,
$$

where $I$ is the $n \times n$ unit matrix and $\lambda_{i}(1 \leq i \leq m-1)$ are complex numbers.

Proof. The proof of this theorem is very easy. By multiplication of formula (2.21) with $A$, we obtain

$$
A F=\frac{1}{\lambda_{m-1}}\left(A^{m}+\lambda_{1} A^{m-1}+\cdots+\lambda_{m-1} A\right) H=O H=0,
$$

or in other words, the function $f \in F$ satisfies the functional equation (2.1) for any $h \in H$.

Conversely, if $F$ is a solution of $A F=\mathbb{O}$, then obviously $F$ satisfies the identity

$$
F=\frac{1}{\lambda_{m-1}}\left(A^{m-1}+\lambda_{1} A^{m-2}+\cdots+\lambda_{m-1} I\right) F,
$$

that is, $F$ can be represented by formula (2.21) with $H=F$. Hence we proved that the function (2.21) is the general solution of (2.1).

EXAMPLE 2.4. The functional equation

$$
2 f\left(\mathbf{Z}_{1}, \mathbf{Z}_{2}, \mathbf{Z}_{3}\right)-3 f\left(\mathbf{Z}_{2}, \mathbf{Z}_{2}, \mathbf{Z}_{3}\right)+f\left(\mathbf{Z}_{3}, \mathbf{Z}_{3}, \mathbf{Z}_{3}\right)=\mathbf{O}
$$


has a matrix of coefficients

$$
A=\left[\begin{array}{rrr}
2 & -3 & 1 \\
0 & -1 & 1 \\
0 & 0 & 0
\end{array}\right]
$$

The matrix $A$ satisfies the following equation:

$$
A^{3}-A^{2}-2 A=O
$$

The required general solution of the above functional equation is

$$
\left[\begin{array}{l}
f\left(\mathbf{Z}_{1}, \mathbf{Z}_{2}, \mathbf{Z}_{3}\right) \\
f\left(\mathbf{Z}_{2}, \mathbf{Z}_{2}, \mathbf{Z}_{3}\right) \\
f\left(\mathbf{Z}_{3}, \mathbf{Z}_{3}, \mathbf{Z}_{3}\right)
\end{array}\right]=-\frac{1}{2}\left(A^{2}-A-2 I\right)\left[\begin{array}{l}
h\left(\mathbf{Z}_{1}, \mathbf{Z}_{2}, \mathbf{Z}_{3}\right) \\
h\left(\mathbf{Z}_{2}, \mathbf{Z}_{2}, \mathbf{Z}_{3}\right) \\
h\left(\mathbf{Z}_{3}, \mathbf{Z}_{3}, \mathbf{Z}_{3}\right)
\end{array}\right],
$$

that is,

$$
f\left(\mathbf{Z}_{1}, \mathbf{Z}_{2}, \mathbf{Z}_{3}\right)=h\left(\mathbf{Z}_{3}, \mathbf{Z}_{3}, \mathbf{Z}_{3}\right) \equiv p\left(\mathbf{Z}_{3}\right)
$$

where $p$ is an arbitrary complex vector function with values in $\mathscr{V}$.

This example shows that Theorem 2.3 can also be applied to equations not of the form (2.1).

THEOREM 2.5. The general solution of the form

$$
\begin{aligned}
f\left(\mathbf{Z}_{1}, \mathbf{Z}_{2}, \ldots, \mathbf{Z}_{n}\right) & =R\left(h\left(\mathbf{Z}_{1}, \mathbf{Z}_{2}, \ldots, \mathbf{Z}_{n}\right)\right) \\
& =\sum_{i=1}^{n} b_{i} h\left(\mathbf{Z}_{i}, \mathbf{Z}_{i+1}, \ldots, \mathbf{Z}_{i+n-1}\right) \quad\left(\mathbf{Z}_{n+i} \equiv \mathbf{Z}_{i}\right)
\end{aligned}
$$

of the functional equation (2.1) is reproductive $(R(R(h))=R(h))$ if and only if the following condition is satisfied:

$$
E(f)=\mathbf{O} \Longrightarrow f=R(f)
$$

Proof. Assume that $R(h)$ is the general solution of the equation $E(f)=\mathbf{O}$. Let $R(R(h))=R(h)$ hold for every $h$. Then, from $E(f)=\mathbf{O}$ it follows that $f=R(h)$ for some $h$, so that for the same $h$ we have $f=R(R(h))=R(f)$.

Conversely, let the condition $E(f)=\mathbf{O} \Rightarrow f=R(f)$ be satisfied. Since for every $h$ it holds that $E(R(h))=\mathbf{O}$, then according to the assumption we obtain $R(h)=R(R(h)$ ) for every $h$.

EXAMPLE 2.6. We will determine the general reproductive solution for the functional equation given in Example 2.2.

On the basis of the general solution, we obtain

$$
\begin{aligned}
R\left(f\left(\mathbf{Z}_{1}, \mathbf{Z}_{2}, \mathbf{Z}_{3}, \mathbf{Z}_{4}\right)\right)= & b_{1} f\left(\mathbf{Z}_{1}, \mathbf{Z}_{2}, \mathbf{Z}_{3}, \mathbf{Z}_{4}\right)+b_{2} f\left(\mathbf{Z}_{2}, \mathbf{Z}_{3}, \mathbf{Z}_{4}, \mathbf{Z}_{1}\right) \\
& +b_{3} f\left(\mathbf{Z}_{3}, \mathbf{Z}_{4}, \mathbf{Z}_{1}, \mathbf{Z}_{2}\right)+\left(b_{1}-b_{2}+b_{3}\right) f\left(\mathbf{Z}_{4}, \mathbf{Z}_{1}, \mathbf{Z}_{2}, \mathbf{Z}_{3}\right)
\end{aligned}
$$




$$
\begin{aligned}
= & b_{1} f\left(\mathbf{Z}_{1}, \mathbf{Z}_{2}, \mathbf{Z}_{3}, \mathbf{Z}_{4}\right)+b_{2} f\left(\mathbf{Z}_{2}, \mathbf{Z}_{3}, \mathbf{Z}_{4}, \mathbf{Z}_{1}\right)+b_{3} f\left(\mathbf{Z}_{3}, \mathbf{Z}_{4}, \mathbf{Z}_{1}, \mathbf{Z}_{2}\right) \\
& +\left(b_{1}-b_{2}+b_{3}\right)\left[f\left(\mathbf{Z}_{1}, \mathbf{Z}_{2}, \mathbf{Z}_{3}, \mathbf{Z}_{4}\right)-f\left(\mathbf{Z}_{2}, \mathbf{Z}_{3}, \mathbf{Z}_{4}, \mathbf{Z}_{1}\right)+f\left(\mathbf{Z}_{3}, \mathbf{Z}_{4}, \mathbf{Z}_{1}, \mathbf{Z}_{2}\right)\right] \\
= & \left(2 b_{1}-b_{2}+b_{3}\right) f\left(\mathbf{Z}_{1}, \mathbf{Z}_{2}, \mathbf{Z}_{3}, \mathbf{Z}_{4}\right)+\left(-b_{1}+2 b_{2}-b_{3}\right) f\left(\mathbf{Z}_{2}, \mathbf{Z}_{3}, \mathbf{Z}_{4}, \mathbf{Z}_{1}\right) \\
& +\left(b_{1}-b_{2}+2 b_{3}\right) f\left(\mathbf{Z}_{3}, \mathbf{Z}_{4}, \mathbf{Z}_{1}, \mathbf{Z}_{2}\right) .
\end{aligned}
$$

The condition $R\left(f\left(\mathbf{Z}_{1}, \mathbf{Z}_{2}, \mathbf{Z}_{3}, \mathbf{Z}_{4}\right)\right)=f\left(\mathbf{Z}_{1}, \mathbf{Z}_{2}, \mathbf{Z}_{3}, \mathbf{Z}_{4}\right)$ holds if

$$
2 b_{1}-b_{2}+b_{3}=1, \quad-b_{1}+2 b_{2}-b_{3}=0, \quad b_{1}-b_{2}+2 b_{3}=0,
$$

that is,

$$
b_{1}=\frac{3}{4}, \quad b_{2}=\frac{1}{4}, \quad b_{3}=-\frac{1}{4} .
$$

Therefore, the general reproductive solution of the given functional equation is

$$
\begin{aligned}
f\left(\mathbf{Z}_{1}, \mathbf{Z}_{2}, \mathbf{Z}_{3}, \mathbf{Z}_{4}\right)= & \frac{3}{4} h\left(\mathbf{Z}_{1}, \mathbf{Z}_{2}, \mathbf{Z}_{3}, \mathbf{Z}_{4}\right)+\frac{1}{4} h\left(\mathbf{Z}_{2}, \mathbf{Z}_{3}, \mathbf{Z}_{4}, \mathbf{Z}_{1}\right) \\
& -\frac{1}{4} h\left(\mathbf{Z}_{3}, \mathbf{Z}_{4}, \mathbf{Z}_{1}, \mathbf{Z}_{2}\right)+\frac{1}{4} h\left(\mathbf{Z}_{4}, \mathbf{Z}_{1}, \mathbf{Z}_{2}, \mathbf{Z}_{3}\right) .
\end{aligned}
$$

Next we will give a procedure by which for every functional equation (2.1) in the case $n=3$ we may determine the canonical equation which is equivalent to it.

For every canonical equation we will determine the general and reproductive solution.

Now we will consider the equation

$$
a_{1} f\left(\mathbf{Z}_{1}, \mathbf{Z}_{2}, \mathbf{Z}_{3}\right)+a_{2} f\left(\mathbf{Z}_{2}, \mathbf{Z}_{3}, \mathbf{Z}_{1}\right)+a_{3} f\left(\mathbf{Z}_{3}, \mathbf{Z}_{1}, \mathbf{Z}_{2}\right)=\mathbf{O} .
$$

From (2.35) by a cyclic permutation of the variables we obtain the following system:

$$
\begin{aligned}
& a_{1} f\left(\mathbf{Z}_{1}, \mathbf{Z}_{2}, \mathbf{Z}_{3}\right)+a_{2} f\left(\mathbf{Z}_{2}, \mathbf{Z}_{3}, \mathbf{Z}_{1}\right)+a_{3} f\left(\mathbf{Z}_{3}, \mathbf{Z}_{1}, \mathbf{Z}_{2}\right)=\mathbf{O}, \\
& a_{3} f\left(\mathbf{Z}_{1}, \mathbf{Z}_{2}, \mathbf{Z}_{3}\right)+a_{1} f\left(\mathbf{Z}_{2}, \mathbf{Z}_{3}, \mathbf{Z}_{1}\right)+a_{2} f\left(\mathbf{Z}_{3}, \mathbf{Z}_{1}, \mathbf{Z}_{2}\right)=\mathbf{O}, \\
& a_{2} f\left(\mathbf{Z}_{1}, \mathbf{Z}_{2}, \mathbf{Z}_{3}\right)+a_{3} f\left(\mathbf{Z}_{2}, \mathbf{Z}_{3}, \mathbf{Z}_{1}\right)+a_{1} f\left(\mathbf{Z}_{3}, \mathbf{Z}_{1}, \mathbf{Z}_{2}\right)=\mathbf{O} .
\end{aligned}
$$

The determinant of system (2.36) is

$$
\Delta=\frac{1}{2}\left(a_{1}+a_{2}+a_{3}\right)\left[\left(a_{1}-a_{2}\right)^{2}+\left(a_{2}-a_{3}\right)^{2}+\left(a_{3}-a_{1}\right)^{2}\right] .
$$

There are four possible cases:
(a) $a_{1}+a_{2}+a_{3} \neq 0$ and $\left(a_{1}-a_{2}\right)^{2}+\left(a_{2}-a_{3}\right)^{2}+\left(a_{3}-a_{1}\right)^{2} \neq 0$,
(b) $a_{1}+a_{2}+a_{3} \neq 0$ and $\left(a_{1}-a_{2}\right)^{2}+\left(a_{2}-a_{3}\right)^{2}+\left(a_{3}-a_{1}\right)^{2}=0$,
(c) $a_{1}+a_{2}+a_{3}=0$ and $\left(a_{1}-a_{2}\right)^{2}+\left(a_{2}-a_{3}\right)^{2}+\left(a_{3}-a_{1}\right)^{2} \neq 0$,
(d) $a_{1}+a_{2}+a_{3}=0$ and $\left(a_{1}-a_{2}\right)^{2}+\left(a_{2}-a_{3}\right)^{2}+\left(a_{3}-a_{1}\right)^{2}=0$.

In the case (a), system (2.36) is obviously equivalent to equation

$$
f\left(\mathbf{Z}_{1}, \mathbf{Z}_{2}, \mathbf{Z}_{3}\right)=\mathbf{O} .
$$


In the case (b), we have

$$
a_{3}^{2}-\left(a_{1}+a_{2}\right) a_{3}+a_{1}^{2}-a_{1} a_{2}+a_{2}^{2}=0
$$

or

$$
a_{3}=\frac{\left[a_{1}+a_{2} \pm i\left(a_{1}-a_{2}\right) \sqrt{3}\right]}{2}
$$

If $a_{2}=a_{1}$, then also $a_{3}=a_{1}$ and $a_{1}+a_{2}+a_{3} \neq 0$ implies $a_{1} \neq 0$. Thus system (2.36) is equivalent to the equation

$$
f\left(\mathbf{Z}_{1}, \mathbf{Z}_{2}, \mathbf{Z}_{3}\right)+f\left(\mathbf{Z}_{2}, \mathbf{Z}_{3}, \mathbf{Z}_{1}\right)+f\left(\mathbf{Z}_{3}, \mathbf{Z}_{1}, \mathbf{Z}_{2}\right)=\mathbf{O} .
$$

In the general case, we can write

$$
a_{3}=\frac{1 \pm i \sqrt{3}}{2} a_{1}+\frac{1 \mp i \sqrt{3}}{2} a_{2}
$$

or, if $\omega_{6}$ and $\omega_{6}^{-1}$ are the primitive 6 th roots of 1 , then $a_{3}=a_{1} \omega_{6}+a_{2} \omega_{6}^{-1}$. Now (2.35) takes the form

$$
a_{1} f\left(\mathbf{Z}_{1}, \mathbf{Z}_{2}, \mathbf{Z}_{3}\right)+a_{2} f\left(\mathbf{Z}_{2}, \mathbf{Z}_{3}, \mathbf{Z}_{1}\right)+\left(a_{1} \omega_{6}+a_{2} \omega_{6}^{-1}\right) f\left(\mathbf{Z}_{3}, \mathbf{Z}_{1}, \mathbf{Z}_{2}\right)=\mathbf{O} .
$$

If $a_{3}=0$, we have $a_{1}+a_{2} \neq 0, a_{1} \omega_{6}^{2}+a_{2}=0$ and the equation takes the form

$$
f\left(\mathbf{Z}_{1}, \mathbf{Z}_{2}, \mathbf{Z}_{3}\right)-\omega_{3} f\left(\mathbf{Z}_{2}, \mathbf{Z}_{3}, \mathbf{Z}_{1}\right)=\mathbf{O},
$$

where $\omega_{3}$ is a primitive third root of 1 (we assume $\omega_{3}=\omega_{6}^{2}$ ).

If $a_{2}=0$ or $a_{1}=0$, we obtain, respectively, the equations

$$
\begin{aligned}
f\left(\mathbf{Z}_{1}, \mathbf{Z}_{2}, \mathbf{Z}_{3}\right)+\omega_{6} f\left(\mathbf{Z}_{3}, \mathbf{Z}_{1}, \mathbf{Z}_{2}\right) & =\mathbf{O}, \\
f\left(\mathbf{Z}_{2}, \mathbf{Z}_{3}, \mathbf{Z}_{1}\right)+\omega_{6}^{-1} f\left(\mathbf{Z}_{3}, \mathbf{Z}_{1}, \mathbf{Z}_{2}\right) & =\mathbf{O},
\end{aligned}
$$

which can be reduced to (2.44) by a cyclic permutation of the vectors.

We will see that (2.43) can always be reduced to (2.41) or (2.44). To this end we will use the following lemma.

LEMMA 2.7. Suppose that (2.35) can be written in the form

$$
\begin{gathered}
\alpha_{1}\left[c_{1} f\left(\mathbf{Z}_{1}, \mathbf{Z}_{2}, \mathbf{Z}_{3}\right)+c_{2} f\left(\mathbf{Z}_{2}, \mathbf{Z}_{3}, \mathbf{Z}_{1}\right)\right]+\alpha_{2}\left[c_{1} f\left(\mathbf{Z}_{2}, \mathbf{Z}_{3}, \mathbf{Z}_{1}\right)+c_{2} f\left(\mathbf{Z}_{3}, \mathbf{Z}_{1}, \mathbf{Z}_{2}\right)\right] \\
+\alpha_{3}\left[c_{1} f\left(\mathbf{Z}_{3}, \mathbf{Z}_{1}, \mathbf{Z}_{2}\right)+c_{2} f\left(\mathbf{Z}_{1}, \mathbf{Z}_{2}, \mathbf{Z}_{3}\right)\right]=\mathbf{O},
\end{gathered}
$$

where

$$
\left|\begin{array}{lll}
\alpha_{1} & \alpha_{2} & \alpha_{3} \\
\alpha_{3} & \alpha_{1} & \alpha_{2} \\
\alpha_{2} & \alpha_{3} & \alpha_{1}
\end{array}\right| \neq 0
$$

Then (2.35) is equivalent to

$$
c_{1} f\left(\mathbf{Z}_{1}, \mathbf{Z}_{2}, \mathbf{Z}_{3}\right)+c_{2} f\left(\mathbf{Z}_{2}, \mathbf{Z}_{3}, \mathbf{Z}_{1}\right)=\mathbf{O} .
$$


Proof. By a cyclic permutation of (2.46) we derive the following system:

$$
\begin{aligned}
& \alpha_{1}\left[c_{1} f\left(\mathbf{Z}_{1}, \mathbf{Z}_{2}, \mathbf{Z}_{3}\right)+c_{2} f\left(\mathbf{Z}_{2}, \mathbf{Z}_{3}, \mathbf{Z}_{1}\right)\right]+\alpha_{2}\left[c_{1} f\left(\mathbf{Z}_{2}, \mathbf{Z}_{3}, \mathbf{Z}_{1}\right)+c_{2} f\left(\mathbf{Z}_{3}, \mathbf{Z}_{1}, \mathbf{Z}_{2}\right)\right] \\
& \quad+\alpha_{3}\left[c_{1} f\left(\mathbf{Z}_{3}, \mathbf{Z}_{1}, \mathbf{Z}_{2}\right)+c_{2} f\left(\mathbf{Z}_{1}, \mathbf{Z}_{2}, \mathbf{Z}_{3}\right)\right]=\mathbf{O}, \\
& \alpha_{3}\left[c_{1} f\left(\mathbf{Z}_{1}, \mathbf{Z}_{2}, \mathbf{Z}_{3}\right)+c_{2} f\left(\mathbf{Z}_{2}, \mathbf{Z}_{3}, \mathbf{Z}_{1}\right)\right]+\alpha_{1}\left[c_{1} f\left(\mathbf{Z}_{2}, \mathbf{Z}_{3}, \mathbf{Z}_{1}\right)+c_{2} f\left(\mathbf{Z}_{3}, \mathbf{Z}_{1}, \mathbf{Z}_{2}\right)\right] \\
& \quad+\alpha_{2}\left[c_{1} f\left(\mathbf{Z}_{3}, \mathbf{Z}_{1}, \mathbf{Z}_{2}\right)+c_{2} f\left(\mathbf{Z}_{1}, \mathbf{Z}_{2}, \mathbf{Z}_{3}\right)\right]=\mathbf{O}, \\
& \alpha_{2}\left[c_{1} f\left(\mathbf{Z}_{1}, \mathbf{Z}_{2}, \mathbf{Z}_{3}\right)+c_{2} f\left(\mathbf{Z}_{2}, \mathbf{Z}_{3}, \mathbf{Z}_{1}\right)\right]+\alpha_{3}\left[c_{1} f\left(\mathbf{Z}_{2}, \mathbf{Z}_{3}, \mathbf{Z}_{1}\right)+c_{2} f\left(\mathbf{Z}_{3}, \mathbf{Z}_{1}, \mathbf{Z}_{2}\right)\right] \\
& \quad+\alpha_{1}\left[c_{1} f\left(\mathbf{Z}_{3}, \mathbf{Z}_{1}, \mathbf{Z}_{2}\right)+c_{2} f\left(\mathbf{Z}_{1}, \mathbf{Z}_{2}, \mathbf{Z}_{3}\right)\right]=\mathbf{O} .
\end{aligned}
$$

The determinant of this system is

$$
\left|\begin{array}{lll}
\alpha_{1} & \alpha_{2} & \alpha_{3} \\
\alpha_{3} & \alpha_{1} & \alpha_{2} \\
\alpha_{2} & \alpha_{3} & \alpha_{1}
\end{array}\right| \neq 0
$$

thus we deduce (2.48).

We see that (2.43) can be written in the form (2.46)

$$
\begin{gathered}
0\left[f\left(\mathbf{Z}_{1}, \mathbf{Z}_{2}, \mathbf{Z}_{3}\right)-\omega_{3} f\left(\mathbf{Z}_{2}, \mathbf{Z}_{3}, \mathbf{Z}_{1}\right)\right]+a_{2}\left[f\left(\mathbf{Z}_{2}, \mathbf{Z}_{3}, \mathbf{Z}_{1}\right)-\omega_{3} f\left(\mathbf{Z}_{3}, \mathbf{Z}_{1}, \mathbf{Z}_{2}\right)\right] \\
+a_{1} \omega_{6}\left[f\left(\mathbf{Z}_{3}, \mathbf{Z}_{1}, \mathbf{Z}_{2}\right)-\omega_{3} f\left(\mathbf{Z}_{1}, \mathbf{Z}_{2}, \mathbf{Z}_{3}\right)\right]=\mathbf{O} .
\end{gathered}
$$

The determinant in condition (2.47) is

$$
\left|\begin{array}{ccc}
0 & a_{2} & a_{1} \omega_{6} \\
a_{1} \omega_{6} & 0 & a_{2} \\
a_{2} & a_{1} \omega_{6} & 0
\end{array}\right|=a_{2}^{3}-a_{1}^{3} .
$$

If $a_{2}^{3} \neq a_{1}^{3}$, then by Lemma 2.7, (2.43) is equivalent to (2.44). If $a_{2}=a_{1}$, then it is equivalent to (2.41). If $a_{2}^{3}=a_{1}^{3}$, but $a_{2} \neq a_{1}$, then $a_{2}=a_{1} \omega_{6}^{2}$ or $a_{2}=a_{1} \omega_{6}^{4}$. In the latter case we have

$$
a_{1}+a_{2}+a_{3}=a_{1}+a_{1} \omega_{6}^{4}+a_{1} \omega_{6}+a_{1} \omega_{6}^{3}=a_{1}-a_{1} \omega_{6}+a_{1} \omega_{6}-a_{1}=0
$$

which is a contradiction. Suppose that $a_{2}=a_{1} \omega_{6}^{2}$. Then $a_{3}=2 a_{1} \omega_{6}$ and (2.43) becomes

$$
f\left(\mathbf{Z}_{1}, \mathbf{Z}_{2}, \mathbf{Z}_{3}\right)+\omega_{6}^{2} f\left(\mathbf{Z}_{2}, \mathbf{Z}_{3}, \mathbf{Z}_{1}\right)+2 \omega_{6} f\left(\mathbf{Z}_{3}, \mathbf{Z}_{1}, \mathbf{Z}_{2}\right)=\mathbf{O}
$$

or

$$
\begin{gathered}
f\left(\mathbf{Z}_{1}, \mathbf{Z}_{2}, \mathbf{Z}_{3}\right)-\omega_{3} f\left(\mathbf{Z}_{2}, \mathbf{Z}_{3}, \mathbf{Z}_{1}\right)+2 \omega_{3}\left[f\left(\mathbf{Z}_{2}, \mathbf{Z}_{3}, \mathbf{Z}_{1}\right)-\omega_{3} f\left(\mathbf{Z}_{3}, \mathbf{Z}_{1}, \mathbf{Z}_{2}\right)\right] \\
+0\left[f\left(\mathbf{Z}_{3}, \mathbf{Z}_{1}, \mathbf{Z}_{2}\right)-\omega_{3} f\left(\mathbf{Z}_{1}, \mathbf{Z}_{2}, \mathbf{Z}_{3}\right)\right]=\mathbf{O} .
\end{gathered}
$$

The determinant

$$
\left|\begin{array}{ccc}
1 & 2 \omega_{3} & 0 \\
0 & 1 & 2 \omega_{3} \\
2 \omega_{3} & 0 & 1
\end{array}\right|=1+8 \omega_{3}^{3}=9 \neq 0
$$

thus by Lemma $2.7,(2.43)$ is equivalent to (2.44). 
In the case (c), $a_{1}+a_{2}+a_{3}=0$ and at least one of the inequalities $a_{1}-a_{2} \neq 0$, $a_{2}-a_{3} \neq 0$ and $a_{3}-a_{1} \neq 0$ is valid. Suppose, for the sake of definiteness, that $a_{1}-a_{2} \neq$ 0 . In view of $a_{3}=-a_{1}-a_{2}$ we have

$$
a_{1} f\left(\mathbf{Z}_{1}, \mathbf{Z}_{2}, \mathbf{Z}_{3}\right)+a_{2} f\left(\mathbf{Z}_{2}, \mathbf{Z}_{3}, \mathbf{Z}_{1}\right)+\left(-a_{1}-a_{2}\right) f\left(\mathbf{Z}_{3}, \mathbf{Z}_{1}, \mathbf{Z}_{2}\right)=\mathbf{O},
$$

or the form (2.46)

$$
\begin{gathered}
0\left[f\left(\mathbf{Z}_{1}, \mathbf{Z}_{2}, \mathbf{Z}_{3}\right)-f\left(\mathbf{Z}_{2}, \mathbf{Z}_{3}, \mathbf{Z}_{1}\right)\right]+a_{2}\left[f\left(\mathbf{Z}_{2}, \mathbf{Z}_{3}, \mathbf{Z}_{1}\right)-f\left(\mathbf{Z}_{3}, \mathbf{Z}_{1}, \mathbf{Z}_{2}\right)\right] \\
-a_{1}\left[f\left(\mathbf{Z}_{3}, \mathbf{Z}_{1}, \mathbf{Z}_{2}\right)-f\left(\mathbf{Z}_{1}, \mathbf{Z}_{2}, \mathbf{Z}_{3}\right)\right]=\mathbf{O} .
\end{gathered}
$$

The determinant in (2.47) is

$$
\left|\begin{array}{rrr}
0 & a_{2} & -a_{1} \\
-a_{1} & 0 & a_{2} \\
a_{2} & -a_{1} & 0
\end{array}\right|=a_{2}^{3}-a_{1}^{3} .
$$

Since $a_{1}-a_{2} \neq 0$, the equality $a_{2}^{3}-a_{1}^{3}=0$ is possible only for $a_{2}=a_{1} \omega_{3}\left(\omega_{3}\right.$ a primitive third root of 1$)$, then $a_{3}=-a_{1}\left(1+\omega_{3}\right)$ and we are led to a contradiction with $\left(a_{1}-a_{2}\right)^{2}+\left(a_{2}-a_{3}\right)^{2}+\left(a_{3}-a_{1}\right)^{2} \neq 0$. Thus, in this case (2.35) is equivalent to the equation

$$
f\left(\mathbf{Z}_{1}, \mathbf{Z}_{2}, \mathbf{Z}_{3}\right)-f\left(\mathbf{Z}_{2}, \mathbf{Z}_{3}, \mathbf{Z}_{1}\right)=\mathbf{O} .
$$

If $a_{1}-a_{2}=0$, then at least one of the differences $a_{2}-a_{3}$ and $a_{3}-a_{1}$ is not 0 and we come to the same conclusion.

In the case (d), we have $a_{3}=-a_{1}-a_{2}, a_{1}^{2}+a_{1} a_{2}+a_{2}^{2}=0$, that is, either $a_{1}=a_{2}=$ $a_{3}=0$ and (2.35) reduces to the identity

$$
\mathbf{O}=\mathbf{O}
$$

or $a_{2}=a_{1} \omega_{3}, a_{3}=a_{1} \omega_{3}^{2}$, where $\omega_{3}$ is as above. Now (2.35) reduces to

$$
f\left(\mathbf{Z}_{1}, \mathbf{Z}_{2}, \mathbf{Z}_{3}\right)+\omega_{3} f\left(\mathbf{Z}_{2}, \mathbf{Z}_{3}, \mathbf{Z}_{1}\right)+\omega_{3}^{2} f\left(\mathbf{Z}_{3}, \mathbf{Z}_{1}, \mathbf{Z}_{2}\right)=\mathbf{O} .
$$

On the basis of the exposition we conclude that the following lemma holds.

LEMMA 2.8. The functional equation (2.35) is equivalent to the following equations: (I) $f\left(\mathbf{Z}_{1}, \mathbf{Z}_{2}, \mathbf{Z}_{3}\right)=\mathbf{O}$ if

$$
a_{1}+a_{2}+a_{3} \neq 0, \quad\left(a_{1}-a_{2}\right)^{2}+\left(a_{2}-a_{3}\right)^{2}+\left(a_{3}-a_{1}\right)^{2} \neq 0 ;
$$

(II) (2.41) or (2.44) if

$$
a_{1}+a_{2}+a_{3} \neq 0, \quad\left(a_{1}-a_{2}\right)^{2}+\left(a_{2}-a_{3}\right)^{2}+\left(a_{3}-a_{1}\right)^{2}=0 ;
$$

(III) (2.60) if

$$
a_{1}+a_{2}+a_{3}=0, \quad\left(a_{1}-a_{2}\right)^{2}+\left(a_{2}-a_{3}\right)^{2}+\left(a_{3}-a_{1}\right)^{2} \neq 0 ;
$$

(IV) $\mathbf{O}=\mathbf{O}$ or (2.62) if

$$
a_{1}+a_{2}+a_{3}=0, \quad\left(a_{1}-a_{2}\right)^{2}+\left(a_{2}-a_{3}\right)^{2}+\left(a_{3}-a_{1}\right)^{2}=0 .
$$

For any of the above equations, we give formulas for the general solutions and formulas for the general reproductive solutions of these equations. 
Proposition 2.9. The equation

$$
f\left(\mathbf{Z}_{1}, \mathbf{Z}_{2}, \mathbf{Z}_{3}\right)+f\left(\mathbf{Z}_{2}, \mathbf{Z}_{3}, \mathbf{Z}_{1}\right)+f\left(\mathbf{Z}_{3}, \mathbf{Z}_{1}, \mathbf{Z}_{2}\right)=\mathbf{O}
$$

has a general solution given by

$$
f\left(\mathbf{Z}_{1}, \mathbf{Z}_{2}, \mathbf{Z}_{3}\right)=b_{1} h\left(\mathbf{Z}_{1}, \mathbf{Z}_{2}, \mathbf{Z}_{3}\right)+b_{2} h\left(\mathbf{Z}_{2}, \mathbf{Z}_{3}, \mathbf{Z}_{1}\right)-\left(b_{1}+b_{2}\right) h\left(\mathbf{Z}_{3}, \mathbf{Z}_{1}, \mathbf{Z}_{2}\right),
$$

where $h$ is an arbitrary complex vector function with values in $\mathscr{V}$ and $b_{1}, b_{2}$ are arbitrary complex constants.

Proof. From the given equation we obtain the system

$$
\begin{aligned}
& f\left(\mathbf{Z}_{1}, \mathbf{Z}_{2}, \mathbf{Z}_{3}\right)+f\left(\mathbf{Z}_{2}, \mathbf{Z}_{3}, \mathbf{Z}_{1}\right)+f\left(\mathbf{Z}_{3}, \mathbf{Z}_{1}, \mathbf{Z}_{2}\right)=\mathbf{O}, \\
& f\left(\mathbf{Z}_{1}, \mathbf{Z}_{2}, \mathbf{Z}_{3}\right)+f\left(\mathbf{Z}_{2}, \mathbf{Z}_{3}, \mathbf{Z}_{1}\right)+f\left(\mathbf{Z}_{3}, \mathbf{Z}_{1}, \mathbf{Z}_{2}\right)=\mathbf{O}, \\
& f\left(\mathbf{Z}_{1}, \mathbf{Z}_{2}, \mathbf{Z}_{3}\right)+f\left(\mathbf{Z}_{2}, \mathbf{Z}_{3}, \mathbf{Z}_{1}\right)+f\left(\mathbf{Z}_{3}, \mathbf{Z}_{1}, \mathbf{Z}_{2}\right)=\mathbf{O},
\end{aligned}
$$

with matrix of coefficients

$$
A=\left[\begin{array}{lll}
1 & 1 & 1 \\
1 & 1 & 1 \\
1 & 1 & 1
\end{array}\right]
$$

The general solution of this system, according to (2.9) is given by

$$
\left[\begin{array}{l}
f\left(\mathbf{Z}_{1}, \mathbf{Z}_{2}, \mathbf{Z}_{3}\right) \\
f\left(\mathbf{Z}_{2}, \mathbf{Z}_{3}, \mathbf{Z}_{1}\right) \\
f\left(\mathbf{Z}_{3}, \mathbf{Z}_{1}, \mathbf{Z}_{2}\right)
\end{array}\right]=B\left[\begin{array}{l}
h\left(\mathbf{Z}_{1}, \mathbf{Z}_{2}, \mathbf{Z}_{3}\right) \\
h\left(\mathbf{Z}_{2}, \mathbf{Z}_{3}, \mathbf{Z}_{1}\right) \\
h\left(\mathbf{Z}_{3}, \mathbf{Z}_{1}, \mathbf{Z}_{2}\right)
\end{array}\right],
$$

where $B$ is a general cyclic $3 \times 3$ matrix which satisfies the condition $A B=O$.

On the basis of formula (2.9), we may write

$$
f\left(\mathbf{Z}_{1}, \mathbf{Z}_{2}, \mathbf{Z}_{3}\right)=b_{1} h\left(\mathbf{Z}_{1}, \mathbf{Z}_{2}, \mathbf{Z}_{3}\right)+b_{2} h\left(\mathbf{Z}_{2}, \mathbf{Z}_{3}, \mathbf{Z}_{1}\right)-\left(b_{1}+b_{2}\right) h\left(\mathbf{Z}_{3}, \mathbf{Z}_{1}, \mathbf{Z}_{2}\right),
$$

which proves the proposition.

Proposition 2.10. The reproductive solution of (2.67) is

$$
f\left(\mathbf{Z}_{1}, \mathbf{Z}_{2}, \mathbf{Z}_{3}\right)=\frac{2}{3} h\left(\mathbf{Z}_{1}, \mathbf{Z}_{2}, \mathbf{Z}_{3}\right)-\frac{1}{3} h\left(\mathbf{Z}_{2}, \mathbf{Z}_{3}, \mathbf{Z}_{1}\right)-\frac{1}{3} h\left(\mathbf{Z}_{3}, \mathbf{Z}_{1}, \mathbf{Z}_{2}\right),
$$

where $h$ is an arbitrary complex vector function with values in $\mathscr{V}$.

Proof. This statement will be proved in the following way. From the general solution (2.68) we obtain

$$
\begin{aligned}
R\left(f\left(\mathbf{Z}_{1}, \mathbf{Z}_{2}, \mathbf{Z}_{3}\right)\right)= & b_{1} f\left(\mathbf{Z}_{1}, \mathbf{Z}_{2}, \mathbf{Z}_{3}\right)+b_{2} f\left(\mathbf{Z}_{2}, \mathbf{Z}_{3}, \mathbf{Z}_{1}\right)-\left(b_{1}+b_{2}\right) f\left(\mathbf{Z}_{3}, \mathbf{Z}_{1}, \mathbf{Z}_{2}\right) \\
= & b_{1} f\left(\mathbf{Z}_{1}, \mathbf{Z}_{2}, \mathbf{Z}_{3}\right)+b_{2} f\left(\mathbf{Z}_{2}, \mathbf{Z}_{3}, \mathbf{Z}_{1}\right) \\
& +\left(b_{1}+b_{2}\right)\left[f\left(\mathbf{Z}_{1}, \mathbf{Z}_{2}, \mathbf{Z}_{3}\right)+f\left(\mathbf{Z}_{2}, \mathbf{Z}_{3}, \mathbf{Z}_{1}\right)\right] \\
= & \left(2 b_{1}+b_{2}\right) f\left(\mathbf{Z}_{1}, \mathbf{Z}_{2}, \mathbf{Z}_{3}\right)+\left(b_{1}+2 b_{2}\right) f\left(\mathbf{Z}_{2}, \mathbf{Z}_{3}, \mathbf{Z}_{1}\right) .
\end{aligned}
$$

The condition $R\left(f\left(\mathbf{Z}_{1}, \mathbf{Z}_{2}, \mathbf{Z}_{3}\right)\right)=f\left(\mathbf{Z}_{1}, \mathbf{Z}_{2}, \mathbf{Z}_{3}\right)$ is satisfied if

$$
b_{1}=\frac{2}{3}, \quad b_{2}=-\frac{1}{3} \text {. }
$$


Thus

$$
f\left(\mathbf{Z}_{1}, \mathbf{Z}_{2}, \mathbf{Z}_{3}\right)=\frac{2}{3} h\left(\mathbf{Z}_{1}, \mathbf{Z}_{2}, \mathbf{Z}_{3}\right)-\frac{1}{3} h\left(\mathbf{Z}_{2}, \mathbf{Z}_{3}, \mathbf{Z}_{1}\right)-\frac{1}{3} h\left(\mathbf{Z}_{3}, \mathbf{Z}_{1}, \mathbf{Z}_{2}\right)
$$

is a reproductive solution of (2.67).

In a similar way, we can prove that (2.44) has a general solution given by

$$
f\left(\mathbf{Z}_{1}, \mathbf{Z}_{2}, \mathbf{Z}_{3}\right)=b\left[h\left(\mathbf{Z}_{1}, \mathbf{Z}_{2}, \mathbf{Z}_{3}\right)+\omega_{3} h\left(\mathbf{Z}_{2}, \mathbf{Z}_{3}, \mathbf{Z}_{1}\right)+\omega_{3}^{2} h\left(\mathbf{Z}_{3}, \mathbf{Z}_{1}, \mathbf{Z}_{2}\right)\right]
$$

where $b$ is a complex constant and $h$ is an arbitrary complex vector function with values in $\mathscr{V}$.

Of course, in the last equality we can put $b=1$, including the arbitrary constant in the function $h$. Also, if we put $b_{1}=b, b_{2}=b \omega_{3}$, then by virtue of the equality $1+\omega_{3}+\omega_{3}^{2}=0$ we see that the general solution of (2.44) is of the form (2.68).

On the other hand, it is sometimes convenient to keep this factor. For instance, we see that the reproductive solution of (2.44) is obtained for $b=1 / 3$, that is,

$$
f\left(\mathbf{Z}_{1}, \mathbf{Z}_{2}, \mathbf{Z}_{3}\right)=\frac{1}{3}\left[h\left(\mathbf{Z}_{1}, \mathbf{Z}_{2}, \mathbf{Z}_{3}\right)+\omega_{3} h\left(\mathbf{Z}_{2}, \mathbf{Z}_{3}, \mathbf{Z}_{1}\right)+\omega_{3}^{2} h\left(\mathbf{Z}_{3}, \mathbf{Z}_{1}, \mathbf{Z}_{2}\right)\right] .
$$

Similarly, (2.60) has a general solution given by

$$
f\left(\mathbf{Z}_{1}, \mathbf{Z}_{2}, \mathbf{Z}_{3}\right)=h\left(\mathbf{Z}_{1}, \mathbf{Z}_{2}, \mathbf{Z}_{3}\right)+h\left(\mathbf{Z}_{2}, \mathbf{Z}_{3}, \mathbf{Z}_{1}\right)+h\left(\mathbf{Z}_{3}, \mathbf{Z}_{1}, \mathbf{Z}_{2}\right)
$$

and a reproductive solution given by

$$
f\left(\mathbf{Z}_{1}, \mathbf{Z}_{2}, \mathbf{Z}_{3}\right)=\frac{1}{3}\left[h\left(\mathbf{Z}_{1}, \mathbf{Z}_{2}, \mathbf{Z}_{3}\right)+h\left(\mathbf{Z}_{2}, \mathbf{Z}_{3}, \mathbf{Z}_{1}\right)+h\left(\mathbf{Z}_{3}, \mathbf{Z}_{1}, \mathbf{Z}_{2}\right)\right]
$$

and (2.62) has a general solution given by

$$
f\left(\mathbf{Z}_{1}, \mathbf{Z}_{2}, \mathbf{Z}_{3}\right)=b_{1} h\left(\mathbf{Z}_{1}, \mathbf{Z}_{2}, \mathbf{Z}_{3}\right)+b_{2} h\left(\mathbf{Z}_{2}, \mathbf{Z}_{3}, \mathbf{Z}_{1}\right)-\left(\omega_{3}^{2} b_{1}+\omega_{3} b_{2}\right) h\left(\mathbf{Z}_{3}, \mathbf{Z}_{1}, \mathbf{Z}_{2}\right)
$$

and a reproductive solution given by

$$
f\left(\mathbf{Z}_{1}, \mathbf{Z}_{2}, \mathbf{Z}_{3}\right)=\frac{2}{3} h\left(\mathbf{Z}_{1}, \mathbf{Z}_{2}, \mathbf{Z}_{3}\right)-\frac{\omega_{3}}{3} h\left(\mathbf{Z}_{2}, \mathbf{Z}_{3}, \mathbf{Z}_{1}\right)-\frac{\omega_{3}^{2}}{3} h\left(\mathbf{Z}_{3}, \mathbf{Z}_{1}, \mathbf{Z}_{2}\right) .
$$

On the basis of the previous results the following two theorems hold.

THEOREM 2.11. The general solution of the equation

$$
a_{1} f\left(\mathbf{Z}_{1}, \mathbf{Z}_{2}, \mathbf{Z}_{3}\right)+a_{2} f\left(\mathbf{Z}_{2}, \mathbf{Z}_{3}, \mathbf{Z}_{1}\right)+a_{3} f\left(\mathbf{Z}_{3}, \mathbf{Z}_{1}, \mathbf{Z}_{2}\right)=\mathbf{O}
$$

is given by the following formulas:

(1) $f\left(\mathbf{Z}_{1}, \mathbf{Z}_{2}, \mathbf{Z}_{3}\right) \equiv \mathbf{O}$ if $a_{1}+a_{2}+a_{3} \neq 0$ and $\left(a_{1}-a_{2}\right)^{2}+\left(a_{2}-a_{3}\right)^{2}+\left(a_{3}-a_{1}\right)^{2} \neq 0$;

(2) $f\left(\mathbf{Z}_{1}, \mathbf{Z}_{2}, \mathbf{Z}_{3}\right)=b_{1} h\left(\mathbf{Z}_{1}, \mathbf{Z}_{2}, \mathbf{Z}_{3}\right)+b_{2} h\left(\mathbf{Z}_{2}, \mathbf{Z}_{3}, \mathbf{Z}_{1}\right)-\left(b_{1}+b_{2}\right) h\left(\mathbf{Z}_{3}, \mathbf{Z}_{1}, \mathbf{Z}_{2}\right)$ if $a_{1}+a_{2}+$ $a_{3} \neq 0$ and $\left(a_{1}-a_{2}\right)^{2}+\left(a_{2}-a_{3}\right)^{2}+\left(a_{3}-a_{1}\right)^{2}=0$, (in particular, $f\left(\mathbf{Z}_{1}, \mathbf{Z}_{2}, \mathbf{Z}_{3}\right)=h\left(\mathbf{Z}_{1}, \mathbf{Z}_{2}, \mathbf{Z}_{3}\right)+\omega_{3} h\left(\mathbf{Z}_{2}, \mathbf{Z}_{3}, \mathbf{Z}_{1}\right)+\omega_{3}^{2} h\left(\mathbf{Z}_{3}, \mathbf{Z}_{1}, \mathbf{Z}_{2}\right)$ if $a_{1}, a_{2}, a_{3}$ are distinct nonzero numbers); 
(3) $f\left(\mathbf{Z}_{1}, \mathbf{Z}_{2}, \mathbf{Z}_{3}\right)=h\left(\mathbf{Z}_{1}, \mathbf{Z}_{2}, \mathbf{Z}_{3}\right)+h\left(\mathbf{Z}_{2}, \mathbf{Z}_{3}, \mathbf{Z}_{1}\right)+h\left(\mathbf{Z}_{3}, \mathbf{Z}_{1}, \mathbf{Z}_{2}\right)$; if $a_{1}+a_{2}+a_{3}=0$ and $\left(a_{1}-a_{2}\right)^{2}+\left(a_{2}-a_{3}\right)^{2}+\left(a_{3}-a_{1}\right)^{2} \neq 0$,

(4) if $a_{1}+a_{2}+a_{3}=0$ and $\left(a_{1}-a_{2}\right)^{2}+\left(a_{2}-a_{3}\right)^{2}+\left(a_{3}-a_{1}\right)^{2}=0$, then

(a) $f\left(\mathbf{Z}_{1}, \mathbf{Z}_{2}, \mathbf{Z}_{3}\right)=h\left(\mathbf{Z}_{1}, \mathbf{Z}_{2}, \mathbf{Z}_{3}\right)$ if $a_{1}=a_{2}=a_{3}=0$;

(b) $f\left(\mathbf{Z}_{1}, \mathbf{Z}_{2}, \mathbf{Z}_{3}\right)=b_{1} h\left(\mathbf{Z}_{1}, \mathbf{Z}_{2}, \mathbf{Z}_{3}\right)+b_{2} h\left(\mathbf{Z}_{2}, \mathbf{Z}_{3}, \mathbf{Z}_{1}\right)-\left(\omega_{3}^{2} b_{1}+\omega_{3} b_{2}\right) h\left(\mathbf{Z}_{3}, \mathbf{Z}_{1}, \mathbf{Z}_{2}\right)$ if $a_{2}=a_{1} \omega_{3}, a_{3}=a_{1} \omega_{3}^{2}, a_{1} \neq 0$, where $h$ is an arbitrary complex vector function with values in $\mathscr{V}$.

THEOREM 2.12. Under the assumptions of the previous theorem denoted by (1)-(4), the general reproductive solutions are given by the following formulas:

(1) $f\left(\mathbf{Z}_{1}, \mathbf{Z}_{2}, \mathbf{Z}_{3}\right) \equiv \mathbf{O}$;

(2) (a) $f\left(\mathbf{Z}_{1}, \mathbf{Z}_{2}, \mathbf{Z}_{3}\right)=(2 / 3) h\left(\mathbf{Z}_{1}, \mathbf{Z}_{2}, \mathbf{Z}_{3}\right)-(1 / 3) h\left(\mathbf{Z}_{2}, \mathbf{Z}_{3}, \mathbf{Z}_{1}\right)-(1 / 3) h\left(\mathbf{Z}_{3}, \mathbf{Z}_{1}, \mathbf{Z}_{2}\right)$ if $a_{1}=a_{2}=a_{3} \neq 0$;

(b) $f\left(\mathbf{Z}_{1}, \mathbf{Z}_{2}, \mathbf{Z}_{3}\right)=(2 / 3) h\left(\mathbf{Z}_{1}, \mathbf{Z}_{2}, \mathbf{Z}_{3}\right)-\left(\omega_{3} / 3\right) h\left(\mathbf{Z}_{2}, \mathbf{Z}_{3}, \mathbf{Z}_{1}\right)-\left(\omega_{3}^{2} / 3\right) h\left(\mathbf{Z}_{3}, \mathbf{Z}_{1}, \mathbf{Z}_{2}\right)$ if $a_{1}, a_{2}, a_{3}$ are distinct;

(3) $f\left(\mathbf{Z}_{1}, \mathbf{Z}_{2}, \mathbf{Z}_{3}\right)=(1 / 3)\left[h\left(\mathbf{Z}_{1}, \mathbf{Z}_{2}, \mathbf{Z}_{3}\right)+h\left(\mathbf{Z}_{2}, \mathbf{Z}_{3}, \mathbf{Z}_{1}\right)+h\left(\mathbf{Z}_{3}, \mathbf{Z}_{1}, \mathbf{Z}_{2}\right)\right]$;

(4) (a) $f\left(\mathbf{Z}_{1}, \mathbf{Z}_{2}, \mathbf{Z}_{3}\right)=h\left(\mathbf{Z}_{1}, \mathbf{Z}_{2}, \mathbf{Z}_{3}\right)$;

(b) $f\left(\mathbf{Z}_{1}, \mathbf{Z}_{2}, \mathbf{Z}_{3}\right)=(2 / 3) h\left(\mathbf{Z}_{1}, \mathbf{Z}_{2}, \mathbf{Z}_{3}\right)-\left(\omega_{3} / 3\right) h\left(\mathbf{Z}_{2}, \mathbf{Z}_{3}, \mathbf{Z}_{1}\right)-\left(\omega_{3}^{2} / 3\right) h\left(\mathbf{Z}_{3}, \mathbf{Z}_{1}, \mathbf{Z}_{2}\right)$.

3. Solution of nonhomogeneous functional equations. Next we will give the following results.

THEOREM 3.1. The basic cyclic complex vector nonhomogeneous functional equation with complex constant coefficients

$$
E(f) \equiv \sum_{i=1}^{n} a_{i} f\left(\mathbf{Z}_{i}, \mathbf{Z}_{i+1}, \ldots, \mathbf{Z}_{i+n-1}\right)=g\left(\mathbf{Z}_{1}, \mathbf{Z}_{2}, \ldots, \mathbf{Z}_{n}\right) \quad\left(\mathbf{Z}_{n+i} \equiv \mathbf{Z}_{i}\right)
$$

where $a_{i}(1 \leq i \leq n)$ are complex constants, has a solution if the right-hand side $g$ satisfies

$$
(A C+I)\left[\begin{array}{c}
g\left(\mathbf{Z}_{1}, \mathbf{Z}_{2}, \ldots, \mathbf{Z}_{n}\right) \\
g\left(\mathbf{Z}_{2}, \mathbf{Z}_{3}, \ldots, \mathbf{Z}_{1}\right) \\
\vdots \\
g\left(\mathbf{Z}_{n}, \mathbf{Z}_{1}, \ldots, \mathbf{Z}_{n-1}\right)
\end{array}\right]=\mathbb{0}
$$

where $A$ is given by (2.3), $C$ is any nonzero $n \times n$ cyclic matrix with complex constant entries satisfying $A C A+A=O, O$ is the $n \times n$ zero matrix, $I$ is the $n \times n$ unit matrix and $O$ is defined as in (2.7).

If (3.2) holds for some $C$, then the general solution of (3.1) is given by the following formula

$$
\left[\begin{array}{c}
f\left(\mathbf{Z}_{1}, \mathbf{Z}_{2}, \ldots, \mathbf{Z}_{n}\right) \\
f\left(\mathbf{Z}_{2}, \mathbf{Z}_{3}, \ldots, \mathbf{Z}_{1}\right) \\
\vdots \\
f\left(\mathbf{Z}_{n}, \mathbf{Z}_{1}, \ldots, \mathbf{Z}_{n-1}\right)
\end{array}\right]=B\left[\begin{array}{c}
h\left(\mathbf{Z}_{1}, \mathbf{Z}_{2}, \ldots, \mathbf{Z}_{n}\right) \\
h\left(\mathbf{Z}_{2}, \mathbf{Z}_{3}, \ldots, \mathbf{Z}_{1}\right) \\
\vdots \\
h\left(\mathbf{Z}_{n}, \mathbf{Z}_{1}, \ldots, \mathbf{Z}_{n-1}\right)
\end{array}\right]-C\left[\begin{array}{c}
g\left(\mathbf{Z}_{1}, \mathbf{Z}_{2}, \ldots, \mathbf{Z}_{n}\right) \\
g\left(\mathbf{Z}_{2}, \mathbf{Z}_{3}, \ldots, \mathbf{Z}_{1}\right) \\
\vdots \\
g\left(\mathbf{Z}_{n}, \mathbf{Z}_{1}, \ldots, \mathbf{Z}_{n-1}\right)
\end{array}\right]
$$


where the nonzero $n \times n$ cyclic matrix B given by (2.3) satisfies the condition

$$
A B=O
$$

and $h$ is an arbitrary complex vector function with values in $\mathcal{V}$.

Proof. By a cyclic permutation of the vectors in (3.1), we get

$$
\begin{aligned}
& a_{1} f\left(\mathbf{Z}_{1}, \mathbf{Z}_{2}, \ldots, \mathbf{Z}_{n}\right)+a_{2} f\left(\mathbf{Z}_{2}, \mathbf{Z}_{3}, \ldots, \mathbf{Z}_{1}\right)+\cdots+a_{n} f\left(\mathbf{Z}_{n}, \mathbf{Z}_{1}, \ldots, \mathbf{Z}_{n-1}\right) \\
& \quad=g\left(\mathbf{Z}_{1}, \mathbf{Z}_{2}, \ldots, \mathbf{Z}_{n}\right), \\
& a_{n} f\left(\mathbf{Z}_{1}, \mathbf{Z}_{2}, \ldots, \mathbf{Z}_{n}\right)+a_{1} f\left(\mathbf{Z}_{2}, \mathbf{Z}_{3}, \ldots, \mathbf{Z}_{1}\right)+\cdots+a_{n-1} f\left(\mathbf{Z}_{n}, \mathbf{Z}_{1}, \ldots, \mathbf{Z}_{n-1}\right) \\
& \quad=g\left(\mathbf{Z}_{2}, \mathbf{Z}_{3}, \ldots, \mathbf{Z}_{1}\right), \\
& \vdots \\
& a_{2} f\left(\mathbf{Z}_{1}, \mathbf{Z}_{2}, \ldots, \mathbf{Z}_{n}\right)+a_{3} f\left(\mathbf{Z}_{2}, \mathbf{Z}_{3}, \ldots, \mathbf{Z}_{1}\right)+\cdots+a_{1} f\left(\mathbf{Z}_{n}, \mathbf{Z}_{1}, \ldots, \mathbf{Z}_{n-1}\right) \\
& \quad=g\left(\mathbf{Z}_{n}, \mathbf{Z}_{1}, \ldots, \mathbf{Z}_{n-1}\right),
\end{aligned}
$$

that is, in a matrix form

$$
A F=G,
$$

where

$$
F=\left[\begin{array}{c}
f\left(\mathbf{Z}_{1}, \mathbf{Z}_{2}, \ldots, \mathbf{Z}_{n}\right) \\
f\left(\mathbf{Z}_{2}, \mathbf{Z}_{3}, \ldots, \mathbf{Z}_{1}\right) \\
\vdots \\
f\left(\mathbf{Z}_{n}, \mathbf{Z}_{1}, \ldots, \mathbf{Z}_{n-1}\right)
\end{array}\right], \quad G=\left[\begin{array}{c}
g\left(\mathbf{Z}_{1}, \mathbf{Z}_{2}, \ldots, \mathbf{Z}_{n}\right) \\
g\left(\mathbf{Z}_{2}, \mathbf{Z}_{3}, \ldots, \mathbf{Z}_{1}\right) \\
\vdots \\
g\left(\mathbf{Z}_{n}, \mathbf{Z}_{1}, \ldots, \mathbf{Z}_{n-1}\right)
\end{array}\right] .
$$

Suppose that (3.6) has a solution $F$ and that $C$ satisfies $A C A+A=O$. Then

$$
(A C+I) G=(A C+I) A F=(A C A+A) F=\mathbb{O},
$$

that is, (3.2) must be satisfied. Conversely, let (3.2) hold for some cyclic matrix $C$. Then $-C G$ is easily seen to be a solution of (3.6)

$$
A(-C G)=-(A C+I) G+I G=I G=G .
$$

Now we prove that (3.3) is the general solution of (3.1).

Let $f$ be a solution of (3.1), which we will write in the form

$$
E(f)=g .
$$

We denote by $f_{h}$ the general solution of the equation $E(f)=\mathbf{O}$, and by $f_{p}$ we denote a particular solution of (3.10).

Then $f=f_{h}+f_{p}$ is the general solution of (3.10). Indeed,

$$
E\left(f_{h}+f_{p}\right)=E\left(f_{h}\right)+E\left(f_{p}\right)=g .
$$

On the other hand, let $f$ be an arbitrary solution of (3.10). Then

$$
E\left(f-f_{p}\right)=E(f)-E\left(f_{p}\right)=g-g=\mathbf{O},
$$


that is, $f-f_{p}$ is a solution of the associated homogeneous equation. So there exists a specialization $\bar{f}_{h}$ of the expression $f_{h}$ such that

$$
f-f_{p}=\bar{f}_{h}, \text { that is, } f=\bar{f}_{h}+f_{p} .
$$

Thus $f_{h}+f_{p}$ includes all solutions of (3.10).

The general solution of the homogeneous equation $E(f)=\mathbf{O}$ given in a matrix form according to Theorem 2.1 is $B H$, where $B$ and $H$ are defined by (2.3) and (2.12), respectively, and a particular solution of the equation $E(f)=g$ in a matrix form is $-C G$, then $F=B H-C G$ includes all solutions of the nonhomogeneous equation.

On the other hand, every function of the form (3.3) satisfies the functional equation (3.1).

Next we will consider the functional equation

$$
a_{1} f\left(\mathbf{Z}_{1}, \mathbf{Z}_{2}, \mathbf{Z}_{3}\right)+a_{2} f\left(\mathbf{Z}_{2}, \mathbf{Z}_{3}, \mathbf{Z}_{1}\right)+a_{3} f\left(\mathbf{Z}_{3}, \mathbf{Z}_{1}, \mathbf{Z}_{2}\right)=g\left(\mathbf{Z}_{1}, \mathbf{Z}_{2}, \mathbf{Z}_{3}\right) .
$$

By a similar procedure as in Section 2 one can prove the following lemma.

LEMMA 3.2. The functional equation (3.14) is equivalent to

(I) $f\left(\mathbf{Z}_{1}, \mathbf{Z}_{2}, \mathbf{Z}_{3}\right)=(1 / \Delta)\left[\left(a_{1}^{2}-a_{2} a_{3}\right) g\left(\mathbf{Z}_{1}, \mathbf{Z}_{2}, \mathbf{Z}_{3}\right)+\left(a_{3}^{2}-a_{1} a_{2}\right) g\left(\mathbf{Z}_{2}, \mathbf{Z}_{3}, \mathbf{Z}_{1}\right)+\left(a_{2}^{2}-\right.\right.$ $\left.\left.a_{1} a_{3}\right) g\left(\mathbf{Z}_{3}, \mathbf{Z}_{1}, \mathbf{Z}_{2}\right)\right]$ if $a_{1}+a_{2}+a_{3} \neq 0$, and $\left(a_{1}-a_{2}\right)^{2}+\left(a_{2}-a_{3}\right)^{2}+\left(a_{3}-\right.$ $\left.a_{1}\right)^{2} \neq 0$;

(II) $f\left(\mathbf{Z}_{1}, \mathbf{Z}_{2}, \mathbf{Z}_{3}\right)+f\left(\mathbf{Z}_{2}, \mathbf{Z}_{3}, \mathbf{Z}_{1}\right)+f\left(\mathbf{Z}_{3}, \mathbf{Z}_{1}, \mathbf{Z}_{2}\right)=\left(1 / a_{1}\right) g\left(\mathbf{Z}_{1}, \mathbf{Z}_{2}, \mathbf{Z}_{3}\right)$ if $a_{1}=a_{2}=$ $a_{3} \neq 0$ or

$$
\begin{aligned}
f\left(\mathbf{Z}_{1}, \mathbf{Z}_{2}, \mathbf{Z}_{3}\right)-\omega_{3} f\left(\mathbf{Z}_{2}, \mathbf{Z}_{3}, \mathbf{Z}_{1}\right) & \\
= & \begin{cases}-\frac{a_{1} a_{2} \omega_{6} g\left(\mathbf{Z}_{1}, \mathbf{Z}_{2}, \mathbf{Z}_{3}\right)+a_{1}^{2} \omega_{6}^{2} g\left(\mathbf{Z}_{2}, \mathbf{Z}_{3}, \mathbf{Z}_{1}\right)+a_{2}^{2} g\left(\mathbf{Z}_{3}, \mathbf{Z}_{1}, \mathbf{Z}_{2}\right)}{a_{2}^{3}-a_{1}^{3}} & \text { if } a_{2}^{3} \neq a_{1}^{3}, \\
\frac{g\left(\mathbf{Z}_{1}, \mathbf{Z}_{2}, \mathbf{Z}_{3}\right)-2 \omega_{3} g\left(\mathbf{Z}_{2}, \mathbf{Z}_{3}, \mathbf{Z}_{1}\right)+4 \omega_{3}^{2} g\left(\mathbf{Z}_{3}, \mathbf{Z}_{1}, \mathbf{Z}_{2}\right)}{9 a_{1}} & \text { if } a_{2}=a_{1} \omega_{3}\end{cases}
\end{aligned}
$$

if $a_{1}+a_{2}+a_{3} \neq 0$, and $\left(a_{1}-a_{2}\right)^{2}+\left(a_{2}-a_{3}\right)^{2}+\left(a_{3}-a_{1}\right)^{2}=0$;

(III) $f\left(\mathbf{Z}_{1}, \mathbf{Z}_{2}, \mathbf{Z}_{3}\right)-f\left(\mathbf{Z}_{2}, \mathbf{Z}_{3}, \mathbf{Z}_{1}\right)=\left(1 /\left(a_{2}^{3}-a_{1}^{3}\right)\right)\left[a_{1} a_{2} g\left(\mathbf{Z}_{1}, \mathbf{Z}_{2}, \mathbf{Z}_{3}\right)+a_{1}^{2} g\left(\mathbf{Z}_{2}, \mathbf{Z}_{3}, \mathbf{Z}_{1}\right)+\right.$ $\left.a_{2}^{2} g\left(\mathbf{Z}_{3}, \mathbf{Z}_{1}, \mathbf{Z}_{2}\right)\right]$ if $a_{1}+a_{2}+a_{3}=0$, and $\left(a_{1}-a_{2}\right)^{2}+\left(a_{2}-a_{3}\right)^{2}+\left(a_{3}-a_{1}\right)^{2} \neq 0$;

(IV) $\mathbf{O}=g\left(\mathbf{Z}_{1}, \mathbf{Z}_{2}, \mathbf{Z}_{3}\right)$ or $f\left(\mathbf{Z}_{1}, \mathbf{Z}_{2}, \mathbf{Z}_{3}\right)+\omega_{3} f\left(\mathbf{Z}_{2}, \mathbf{Z}_{3}, \mathbf{Z}_{1}\right)+\omega_{3}^{2} f\left(\mathbf{Z}_{3}, \mathbf{Z}_{1}, \mathbf{Z}_{2}\right)=\left(1 / a_{1}\right) \times$ $g\left(\mathbf{Z}_{1}, \mathbf{Z}_{2}, \mathbf{Z}_{3}\right)$ if $a_{1}+a_{2}+a_{3}=0$, and $\left(a_{1}-a_{2}\right)^{2}+\left(a_{2}-a_{3}\right)^{2}+\left(a_{3}-a_{1}\right)^{2}=0$.

For each of the above equations we will determine the conditions which must be satisfied by the function $g$ so that the equation should have a solution.

Proposition 3.3. Equation (3.14) whose coefficients satisfy the conditions

$$
a_{1}=a_{2}=a_{3} \neq 0
$$

has a solution if and only if the function $g$ satisfies the condition

$$
g\left(\mathbf{Z}_{1}, \mathbf{Z}_{2}, \mathbf{Z}_{3}\right)-g\left(\mathbf{Z}_{2}, \mathbf{Z}_{3}, \mathbf{Z}_{1}\right)=\mathbf{O} .
$$

Proof. In this case the equation considered is equivalent to the equation

$$
a_{1}\left[f\left(\mathbf{Z}_{1}, \mathbf{Z}_{2}, \mathbf{Z}_{3}\right)+f\left(\mathbf{Z}_{2}, \mathbf{Z}_{3}, \mathbf{Z}_{1}\right)+f\left(\mathbf{Z}_{3}, \mathbf{Z}_{1}, \mathbf{Z}_{2}\right)\right]=g\left(\mathbf{Z}_{1}, \mathbf{Z}_{2}, \mathbf{Z}_{3}\right) .
$$


Since

$$
A=a_{1}\left[\begin{array}{lll}
1 & 1 & 1 \\
1 & 1 & 1 \\
1 & 1 & 1
\end{array}\right],
$$

then $A^{2}=3 a_{1} A$, that is, $A\left(-I / 3 a_{1}\right) A+A=O$, and hence we obtain $C=-I / 3 a_{1}$. The condition $A C G+G=0$ reduces to

$$
\frac{2}{3} g\left(\mathbf{Z}_{1}, \mathbf{Z}_{2}, \mathbf{Z}_{3}\right)-\frac{1}{3} g\left(\mathbf{Z}_{2}, \mathbf{Z}_{3}, \mathbf{Z}_{1}\right)-\frac{1}{3} g\left(\mathbf{Z}_{3}, \mathbf{Z}_{1}, \mathbf{Z}_{2}\right)=\mathbf{O}
$$

and (see Lemma 2.7) eventually to (3.17).

In a similar way, we obtain the necessary and sufficient conditions for solvability of the other equations. Thus, we obtain the following result.

THEOREM 3.4. The functional equation (3.14) has a solution if and only if the function $g$ satisfies the following conditions

(1) $g\left(\mathbf{Z}_{1}, \mathbf{Z}_{2}, \mathbf{Z}_{3}\right)$ is arbitrary if $a_{1}+a_{2}+a_{3} \neq 0,\left(a_{1}-a_{2}\right)^{2}+\left(a_{2}-a_{3}\right)^{2}+\left(a_{3}-\right.$ $\left.a_{1}\right)^{2} \neq 0$;

(2) $g\left(\mathbf{Z}_{1}, \mathbf{Z}_{2}, \mathbf{Z}_{3}\right)-g\left(\mathbf{Z}_{2}, \mathbf{Z}_{3}, \mathbf{Z}_{1}\right)=\mathbf{O}$ or $g\left(\mathbf{Z}_{1}, \mathbf{Z}_{2}, \mathbf{Z}_{3}\right)+\omega_{3} g\left(\mathbf{Z}_{2}, \mathbf{Z}_{3}, \mathbf{Z}_{1}\right)+\omega_{3}^{2} g\left(\mathbf{Z}_{3}\right.$, $\left.\mathbf{Z}_{1}, \mathbf{Z}_{2}\right)=\mathbf{O}$ if $a_{1}+a_{2}+a_{3} \neq 0$, and $\left(a_{1}-a_{2}\right)^{2}+\left(a_{2}-a_{3}\right)^{2}+\left(a_{3}-a_{1}\right)^{2}=0$;

(3) $g\left(\mathbf{Z}_{1}, \mathbf{Z}_{2}, \mathbf{Z}_{3}\right)+g\left(\mathbf{Z}_{2}, \mathbf{Z}_{3}, \mathbf{Z}_{1}\right)+g\left(\mathbf{Z}_{3}, \mathbf{Z}_{1}, \mathbf{Z}_{2}\right)=\mathbf{O}$ if $a_{1}+a_{2}+a_{3}=0,\left(a_{1}-a_{2}\right)^{2}+$ $\left(a_{2}-a_{3}\right)^{2}+\left(a_{3}-a_{1}\right)^{2} \neq 0$

(4) $g=\mathbf{O}$ or $g\left(\mathbf{Z}_{1}, \mathbf{Z}_{2}, \mathbf{Z}_{3}\right)-\omega_{3} g\left(\mathbf{Z}_{2}, \mathbf{Z}_{3}, \mathbf{Z}_{1}\right)=\mathbf{O}$ if $a_{1}+a_{2}+a_{3}=0,\left(a_{1}-a_{2}\right)^{2}+\left(a_{2}-\right.$ $\left.a_{3}\right)^{2}+\left(a_{3}-a_{1}\right)^{2}=0$.

Now we will find the general solution for any equation of Lemma 3.2. We will illustrate this only for the second equation.

Proposition 3.5. The general solution of the equation

$$
f\left(\mathbf{Z}_{1}, \mathbf{Z}_{2}, \mathbf{Z}_{3}\right)+f\left(\mathbf{Z}_{2}, \mathbf{Z}_{3}, \mathbf{Z}_{1}\right)+f\left(\mathbf{Z}_{3}, \mathbf{Z}_{1}, \mathbf{Z}_{2}\right)=\frac{1}{a_{1}} g\left(\mathbf{Z}_{1}, \mathbf{Z}_{2}, \mathbf{Z}_{3}\right)
$$

is given by

$$
\begin{aligned}
f\left(\mathbf{Z}_{1}, \mathbf{Z}_{2}, \mathbf{Z}_{3}\right)= & b_{1} h\left(\mathbf{Z}_{1}, \mathbf{Z}_{2}, \mathbf{Z}_{3}\right)+b_{2} h\left(\mathbf{Z}_{2}, \mathbf{Z}_{3}, \mathbf{Z}_{1}\right) \\
& -\left(b_{1}+b_{2}\right) h\left(\mathbf{Z}_{3}, \mathbf{Z}_{1}, \mathbf{Z}_{2}\right)+\frac{1}{3 a_{1}} g\left(\mathbf{Z}_{1}, \mathbf{Z}_{2}, \mathbf{Z}_{3}\right),
\end{aligned}
$$

where $h$ is an arbitrary complex vector function with values in $\mathcal{V}$.

Proof. The general solution of the corresponding homogeneous equation is

$$
f\left(\mathbf{Z}_{1}, \mathbf{Z}_{2}, \mathbf{Z}_{3}\right)=b_{1} h\left(\mathbf{Z}_{1}, \mathbf{Z}_{2}, \mathbf{Z}_{3}\right)+b_{2} h\left(\mathbf{Z}_{2}, \mathbf{Z}_{3}, \mathbf{Z}_{1}\right)-\left(b_{1}+b_{2}\right) h\left(\mathbf{Z}_{3}, \mathbf{Z}_{1}, \mathbf{Z}_{2}\right) .
$$

The particular solution of the considered nonhomogeneous equation is

$$
f\left(\mathbf{Z}_{1}, \mathbf{Z}_{2}, \mathbf{Z}_{3}\right)=\frac{1}{3 a_{1}} g\left(\mathbf{Z}_{1}, \mathbf{Z}_{2}, \mathbf{Z}_{3}\right) .
$$


Thus, the general solution of the given nonhomogeneous equation is

$$
\begin{aligned}
f\left(\mathbf{Z}_{1}, \mathbf{Z}_{2}, \mathbf{Z}_{3}\right)= & b_{1} h\left(\mathbf{Z}_{1}, \mathbf{Z}_{2}, \mathbf{Z}_{3}\right)+b_{2} h\left(\mathbf{Z}_{2}, \mathbf{Z}_{3}, \mathbf{Z}_{1}\right) \\
& -\left(b_{1}+b_{2}\right) h\left(\mathbf{Z}_{3}, \mathbf{Z}_{1}, \mathbf{Z}_{2}\right)+\frac{1}{3 a_{1}} g\left(\mathbf{Z}_{1}, \mathbf{Z}_{2}, \mathbf{Z}_{3}\right) .
\end{aligned}
$$

TheOREM 3.6. The general solution of (3.14) in the cases given in Theorem 3.4 is

(1) $f\left(\mathbf{Z}_{1}, \mathbf{Z}_{2}, \mathbf{Z}_{3}\right)=(1 / \Delta)\left[\left(a_{1}^{2}-a_{2} a_{3}\right) g\left(\mathbf{Z}_{1}, \mathbf{Z}_{2}, \mathbf{Z}_{3}\right)+\left(a_{3}^{2}-a_{1} a_{2}\right) g\left(\mathbf{Z}_{2}, \mathbf{Z}_{3}, \mathbf{Z}_{1}\right)+\left(a_{2}^{2}-\right.\right.$ $\left.\left.a_{1} a_{3}\right) g\left(\mathbf{Z}_{3}, \mathbf{Z}_{1}, \mathbf{Z}_{2}\right)\right]$ if $a_{1}+a_{2}+a_{3} \neq 0$, and $\left(a_{1}-a_{2}\right)^{2}+\left(a_{2}-a_{3}\right)^{2}+\left(a_{3}-a_{1}\right)^{2} \neq 0$;

(2) $f\left(\mathbf{Z}_{1}, \mathbf{Z}_{2}, \mathbf{Z}_{3}\right)=b_{1} h\left(\mathbf{Z}_{1}, \mathbf{Z}_{2}, \mathbf{Z}_{3}\right)+b_{2} h\left(\mathbf{Z}_{2}, \mathbf{Z}_{3}, \mathbf{Z}_{1}\right)-\left(b_{1}+b_{2}\right) h\left(\mathbf{Z}_{3}, \mathbf{Z}_{1}, \mathbf{Z}_{2}\right)+$ $\left(1 / 3 a_{1}\right) g\left(\mathbf{Z}_{1}, \mathbf{Z}_{2}, \mathbf{Z}_{3}\right)$ if $a_{1}=a_{2}=a_{3} \neq 0$ or

$$
\begin{aligned}
f\left(\mathbf{Z}_{1}, \mathbf{Z}_{2}, \mathbf{Z}_{3}\right) & \\
= & h\left(\mathbf{Z}_{1}, \mathbf{Z}_{2}, \mathbf{Z}_{3}\right)+\omega_{3} h\left(\mathbf{Z}_{2}, \mathbf{Z}_{3}, \mathbf{Z}_{1}\right)+\omega_{3}^{2} h\left(\mathbf{Z}_{3}, \mathbf{Z}_{1}, \mathbf{Z}_{2}\right) \\
& + \begin{cases}\frac{\left(a_{1}^{2}-2 a_{1} a_{2} \omega_{6}-a_{2}^{2} \omega_{6}^{2}\right) g\left(\mathbf{Z}_{1}, \mathbf{Z}_{2}, \mathbf{Z}_{3}\right)+\omega_{3}\left(a_{1}^{2}-a_{1} a_{2} \omega_{6}-2 a_{2}^{2} \omega_{6}^{2}\right) g\left(\mathbf{Z}_{2}, \mathbf{Z}_{3}, \mathbf{Z}_{1}\right)}{3\left(a_{2}^{3}-a_{1}^{3}\right)} & \text { if } a_{2}^{3} \neq a_{1}^{3}, \\
-\frac{\omega_{3}}{3 a_{1}} g\left(\mathbf{Z}_{2}, \mathbf{Z}_{3}, \mathbf{Z}_{1}\right) & \text { if } a_{2}=a_{1} \omega_{3}\end{cases}
\end{aligned}
$$

if $a_{1}+a_{2}+a_{3} \neq 0$, and $\left(a_{1}-a_{2}\right)^{2}+\left(a_{2}-a_{3}\right)^{2}+\left(a_{3}-a_{1}\right)^{2}=0$;

(3) $f\left(\mathbf{Z}_{1}, \mathbf{Z}_{2}, \mathbf{Z}_{3}\right)=b_{1}\left[h\left(\mathbf{Z}_{1}, \mathbf{Z}_{2}, \mathbf{Z}_{3}\right)+h\left(\mathbf{Z}_{2}, \mathbf{Z}_{3}, \mathbf{Z}_{1}\right)+h\left(\mathbf{Z}_{3}, \mathbf{Z}_{1}, \mathbf{Z}_{2}\right)\right]+\left(\left(a_{2}-a_{1}\right) g\left(\mathbf{Z}_{1}\right.\right.$, $\left.\left.\mathbf{Z}_{2}, \mathbf{Z}_{3}\right)+\left(a_{1}+2 a_{2}\right) g\left(\mathbf{Z}_{2}, \mathbf{Z}_{3}, \mathbf{Z}_{1}\right)\right) / 3\left(a_{1}^{2}+a_{1} a_{2}+a_{2}^{2}\right)$ if $a_{1}+a_{2}+a_{3}=0$, and $\left(a_{1}-\right.$ $\left.a_{2}\right)^{2}+\left(a_{2}-a_{3}\right)^{2}+\left(a_{3}-a_{1}\right)^{2} \neq 0$;

(4) $f\left(\mathbf{Z}_{1}, \mathbf{Z}_{2}, \mathbf{Z}_{3}\right)=h\left(\mathbf{Z}_{1}, \mathbf{Z}_{2}, \mathbf{Z}_{3}\right)$ or $f\left(\mathbf{Z}_{1}, \mathbf{Z}_{2}, \mathbf{Z}_{3}\right)=b_{1} h\left(\mathbf{Z}_{1}, \mathbf{Z}_{2}, \mathbf{Z}_{3}\right)+b_{2} h\left(\mathbf{Z}_{2}, \mathbf{Z}_{3}, \mathbf{Z}_{1}\right)-$ $\left(\omega_{3}^{2} b_{1}+\omega_{3} b_{2}\right) h\left(\mathbf{Z}_{3}, \mathbf{Z}_{1}, \mathbf{Z}_{2}\right)+\left(1 / 3 a_{1}\right) g\left(\mathbf{Z}_{1}, \mathbf{Z}_{2}, \mathbf{Z}_{3}\right)$ if $a_{1}+a_{2}+a_{3}=0$, and $\left(a_{1}-\right.$ $\left.a_{2}\right)^{2}+\left(a_{2}-a_{3}\right)^{2}+\left(a_{3}-a_{1}\right)^{2}=0$.

4. Solution of paracyclic functional equations. Let $\mathscr{V}$ be a complex vector space with complex dimension $n$, and let the complex vectors $\mathbf{X}_{i}, \mathbf{Y}_{j} \in \mathscr{V}(1 \leq i, j \leq n)$ be given as above. Throughout this section, $\mathscr{C}_{i}$ are constant complex vectors in $\mathscr{V}$ and let $f: \mathscr{V}^{n+k} \mapsto \mathscr{V}$.

Now we will consider the following paracyclic complex vector functional equation of the first kind

$$
\sum_{i=1}^{n} a_{i} f\left(\mathbf{X}_{i}, \mathbf{X}_{i+1}, \ldots, \mathbf{X}_{i+n-1}, \mathbf{Y}_{i}, \mathbf{Y}_{i+1}, \ldots, \mathbf{Y}_{i+k-1}\right)=\mathbf{O} \quad\left(\mathbf{X}_{n+i} \equiv \mathbf{X}_{i}, \mathbf{Y}_{n+i} \equiv \mathbf{Y}_{i}\right),
$$

where $a_{i}(1 \leq i \leq n)$ are complex constants.

First, we will consider two particular cases for $k=1(n>1)$ and $k=n$.

We determine the general solution of the equation

$$
\sum_{i=1}^{n} a_{i} f\left(\mathbf{X}_{i}, \mathbf{X}_{i+1}, \ldots, \mathbf{X}_{i+n-1}, \mathbf{Y}_{i}\right)=\mathbf{O} .
$$

By a cyclic permutation of the vectors in (4.2), we obtain the matrix system

$$
A F=0 \text {, }
$$


where

$$
A=\left[\begin{array}{cccc}
a_{1} & a_{2} & \cdots & a_{n} \\
a_{n} & a_{1} & \cdots & a_{n-1} \\
\vdots & & & \\
a_{2} & a_{3} & \cdots & a_{1}
\end{array}\right], \quad F=\left[\begin{array}{c}
f\left(\mathbf{X}_{1}, \ldots, \mathbf{X}_{n}, \mathbf{Y}_{1}\right) \\
f\left(\mathbf{X}_{2}, \ldots, \mathbf{X}_{1}, \mathbf{Y}_{2}\right) \\
\vdots \\
f\left(\mathbf{X}_{n}, \ldots, \mathbf{X}_{n-1}, \mathbf{Y}_{n}\right)
\end{array}\right], \quad 0=\left[\begin{array}{c}
\mathbf{O} \\
\mathbf{O} \\
\vdots \\
\mathbf{O}
\end{array}\right]
$$

For system (4.3) the following theorem holds.

THEOREM 4.1. The general solution of the functional equation (4.2) is given by formula

$$
F=B H,
$$

if $A B=O$, where $A$ and $B$ are nonzero $n \times n$ cyclic matrices given by (2.3), $O$ is the $n \times n$ zero matrix and

$$
H=\left[\begin{array}{c}
h\left(\mathbf{X}_{1}, \mathbf{X}_{2}, \ldots, \mathbf{X}_{n}\right) \\
h\left(\mathbf{X}_{2}, \mathbf{X}_{3}, \ldots, \mathbf{X}_{1}\right) \\
\vdots \\
h\left(\mathbf{X}_{n}, \mathbf{X}_{1}, \ldots, \mathbf{X}_{n-1}\right)
\end{array}\right]
$$

where $h$ is an arbitrary complex vector function with values in $\mathcal{V}$.

Proof. If not all coefficients $a_{i}(1 \leq i \leq n)$ are 0 , we can suppose, without loss of generality, that $a_{1} \neq 0$. Then (4.2) is equivalent to the equation

$$
f\left(\mathbf{X}_{1}, \ldots, \mathbf{X}_{n}, \mathbf{Y}_{1}\right)=-\frac{a_{2}}{a_{1}} f\left(\mathbf{X}_{2}, \ldots, \mathbf{X}_{n}, \mathbf{X}_{1}, \mathbf{Y}_{2}\right)-\cdots-\frac{a_{n}}{a_{1}} f\left(\mathbf{X}_{n}, \mathbf{X}_{1}, \ldots, \mathbf{X}_{n-1}, \mathbf{Y}_{n}\right) .
$$

By putting $\mathbf{Y}_{i}=\mathscr{b}_{i}(2 \leq i \leq n)$, where $\mathscr{C}_{i}$ are arbitrary complex constant vectors from $\mathscr{V}$, we obtain

$$
f\left(\mathbf{X}_{1}, \ldots, \mathbf{X}_{n}, \mathbf{Y}_{1}\right)=-\frac{a_{2}}{a_{1}} f\left(\mathbf{X}_{2}, \ldots, \mathbf{X}_{n}, \mathbf{X}_{1}, \mathscr{b}_{2}\right)-\cdots-\frac{a_{n}}{a_{1}} f\left(\mathbf{X}_{n}, \mathbf{X}_{1}, \ldots, \mathbf{X}_{n-1}, \mathscr{b}_{n}\right) .
$$

The right-hand side of the last equation depends on $\mathbf{X}_{1}, \ldots, \mathbf{X}_{n}$ only. Denote this expression by $h\left(\mathbf{X}_{1}, \ldots, \mathbf{X}_{n}\right)$.

Therefore, (4.8) obtains the following form:

$$
f\left(\mathbf{X}_{1}, \ldots, \mathbf{X}_{n}, \mathbf{Y}_{1}\right)=h\left(\mathbf{X}_{1}, \ldots, \mathbf{X}_{n}\right) .
$$

Formula (4.9) is the general solution of (4.2) if and only if it holds that

$$
\sum_{i=1}^{n} a_{i} h\left(\mathbf{X}_{i}, \mathbf{X}_{i+1}, \ldots, \mathbf{X}_{i+n-1}\right)=\mathbf{O} .
$$

The above equation is equivalent to the functional equation (2.1), and therefore Theorem 2.1 holds. Thus (4.5) is true. 
Now, we will solve the functional equation (4.1) if $k=n$.

By denoting the pairs $\left(\mathbf{X}_{i}, \mathbf{Y}_{i}\right)=\mathbf{Z}_{i}(1 \leq i \leq n)$, the functional equation (4.1) takes the form (2.1), then Theorem 2.1 holds, that is, the general solution is given by $F=B H$, where

$$
\begin{gathered}
F=\left[\begin{array}{c}
f\left(\mathbf{Z}_{1}, \mathbf{Z}_{2}, \ldots, \mathbf{Z}_{n}\right) \\
f\left(\mathbf{Z}_{2}, \mathbf{Z}_{3}, \ldots, \mathbf{Z}_{1}\right) \\
\vdots \\
f\left(\mathbf{Z}_{n}, \mathbf{Z}_{1}, \ldots, \mathbf{Z}_{n-1}\right)
\end{array}\right]=\left[\begin{array}{c}
f\left(\mathbf{X}_{1}, \mathbf{X}_{2}, \ldots, \mathbf{X}_{n}, \mathbf{Y}_{1}, \mathbf{Y}_{2}, \ldots, \mathbf{Y}_{n}\right) \\
f\left(\mathbf{X}_{2}, \mathbf{X}_{3}, \ldots, \mathbf{X}_{1}, \mathbf{Y}_{2}, \mathbf{Y}_{3}, \ldots, \mathbf{Y}_{1}\right) \\
\vdots \\
f\left(\mathbf{X}_{n}, \mathbf{X}_{1}, \ldots, \mathbf{X}_{n-1}, \mathbf{Y}_{n}, \mathbf{Y}_{1}, \ldots, \mathbf{Y}_{n-1}\right)
\end{array}\right], \\
H=\left[\begin{array}{c}
h\left(\mathbf{X}_{1}, \mathbf{X}_{2}, \ldots, \mathbf{X}_{n}, \mathbf{Y}_{1}, \mathbf{Y}_{2}, \ldots, \mathbf{Y}_{n}\right) \\
h\left(\mathbf{X}_{2}, \mathbf{X}_{3}, \ldots, \mathbf{X}_{1}, \mathbf{Y}_{2}, \mathbf{Y}_{3}, \ldots, \mathbf{Y}_{1}\right) \\
\vdots \\
h\left(\mathbf{X}_{n}, \mathbf{X}_{1}, \ldots, \mathbf{X}_{n-1}, \mathbf{Y}_{n}, \mathbf{Y}_{1}, \ldots, \mathbf{Y}_{n-1}\right)
\end{array}\right],
\end{gathered}
$$

and $B$ is given by (2.3).

Next, we will consider the case $1<k<n$. To this end, instead of (4.1) we will consider the equation

$$
\begin{aligned}
a_{1} f & \left.\mathbf{X}_{1}, \ldots, \mathbf{X}_{n}, \mathbf{Y}_{1}, \ldots, \mathbf{Y}_{k}, \mathbf{Y}_{k+1}, \ldots, \mathbf{Y}_{n}\right) \\
& +a_{2} f\left(\mathbf{X}_{2}, \ldots, \mathbf{X}_{n}, \mathbf{X}_{1}, \mathbf{Y}_{2}, \ldots, \mathbf{Y}_{k}, \mathbf{Y}_{k+1}, \ldots, \mathbf{Y}_{n}, \mathbf{Y}_{1}\right)+\cdots \\
& +a_{n} f\left(\mathbf{X}_{n}, \mathbf{X}_{1}, \ldots, \mathbf{X}_{n-1}, \mathbf{Y}_{n}, \mathbf{Y}_{1}, \ldots, \mathbf{Y}_{n+k-1}, \mathbf{Y}_{n+k}, \ldots, \mathbf{Y}_{n-1}\right)=\mathbf{O} .
\end{aligned}
$$

By a cyclic permutation of the vectors in the last equation, we obtain the matrix system (4.3), where

$$
F=\left[\begin{array}{c}
f\left(\mathbf{X}_{1}, \ldots, \mathbf{X}_{n}, \mathbf{Y}_{1}, \ldots, \mathbf{Y}_{k}, \mathbf{Y}_{k+1}, \ldots, \mathbf{Y}_{n}\right) \\
f\left(\mathbf{X}_{2}, \ldots, \mathbf{X}_{n}, \mathbf{X}_{1}, \mathbf{Y}_{2}, \ldots, \mathbf{Y}_{k}, \mathbf{Y}_{k+1}, \ldots, \mathbf{Y}_{n}, \mathbf{Y}_{1}\right) \\
\vdots \\
f\left(\mathbf{X}_{n}, \mathbf{X}_{1}, \ldots, \mathbf{X}_{n-1}, \mathbf{Y}_{n}, \mathbf{Y}_{1}, \ldots, \mathbf{Y}_{n+k-1}, \mathbf{Y}_{n+k}, \ldots, \mathbf{Y}_{n-1}\right)
\end{array}\right],
$$

$A$ and 0 are as in (4.4).

The necessary and sufficient condition for system (4.3) with (4.13) to have nontrivial solution is $\operatorname{det} A=0$. Since $\operatorname{det} A$ is cyclic, then its value is

$$
\operatorname{det} A=\prod_{i=0}^{n-1} E\left(\varepsilon_{i}\right),
$$

where $\varepsilon_{i}(0 \leq i \leq n-1)$ are distinct roots of the binomial equation

$$
b(x) \equiv 1-x^{n}=0 .
$$

Therefore, (4.12) has nontrivial solutions if and only if the characteristic equation $E(x) \equiv a_{1}+a_{2} x+\cdots+a_{n} x^{n-1}=0$ has common roots with the binomial equation $b(x) \equiv 1-x^{n}=0$. If this is so, we can write $E(x)=P(x) D(x), b(x)=D(x) F(x)$, where $D(x)$ is the greatest common divisor of the polynomials $E(x)$ and $b(x)$. 
The general solution of (4.12) is given by formula

$$
\begin{aligned}
& f\left(\mathbf{X}_{1}, \ldots, \mathbf{X}_{n}, \mathbf{Y}_{1}, \ldots, \mathbf{Y}_{k}, \mathbf{Y}_{k+1}, \ldots, \mathbf{Y}_{n}\right) \\
&=b_{1} h\left(\mathbf{X}_{1}, \ldots, \mathbf{X}_{n}, \mathbf{Y}_{1}, \ldots, \mathbf{Y}_{m}, \mathbf{Y}_{m+1}, \ldots, \mathbf{Y}_{n}\right) \\
&+b_{2} h\left(\mathbf{X}_{2}, \ldots, \mathbf{X}_{n}, \mathbf{X}_{1}, \mathbf{Y}_{2}, \ldots, \mathbf{Y}_{m}, \mathbf{Y}_{m+1}, \ldots, \mathbf{Y}_{n}, \mathbf{Y}_{1}\right)+\cdots \\
&+b_{s+1} h\left(\mathbf{X}_{s+1}, \ldots, \mathbf{X}_{n}, \mathbf{X}_{1}, \ldots, \mathbf{X}_{s}, \mathbf{Y}_{s+1}, \ldots, \mathbf{Y}_{m+s}, \mathbf{Y}_{m+s+1}, \ldots, \mathbf{Y}_{n}, \mathbf{Y}_{1}, \ldots, \mathbf{Y}_{s}\right),
\end{aligned}
$$

where the complex numbers $b_{i}(1 \leq i \leq s+1)$ are coefficients of the polynomial

$$
b_{1}+b_{2} x+\cdots+b_{s+1} x^{s}=F(x)
$$

The functional equation (4.1) will be called reduced equation of (4.12). Now we will prove the following result.

THEOREM 4.2. Every function $f$ given by

$$
\begin{aligned}
f\left(\mathbf{X}_{1}, \ldots, \mathbf{X}_{n}, \mathbf{Y}_{1}, \ldots, \mathbf{Y}_{n}\right)= & b_{1} h\left(\mathbf{X}_{1}, \ldots, \mathbf{X}_{n}, \mathbf{Y}_{1}, \ldots, \mathbf{Y}_{m}\right) \\
& +b_{2} h\left(\mathbf{X}_{2}, \ldots, \mathbf{X}_{n}, \mathbf{X}_{1}, \mathbf{Y}_{2}, \ldots, \mathbf{Y}_{m+1}\right) \\
& +\cdots+b_{s+1} h\left(\mathbf{X}_{s+1}, \ldots, \mathbf{X}_{n}, \mathbf{X}_{1}, \ldots, \mathbf{X}_{s}, \mathbf{Y}_{s+1}, \ldots, \mathbf{Y}_{m+s}\right),
\end{aligned}
$$

satisfies (4.1), where $m=k-s$ for $k>s, h\left(\mathbf{X}_{1}, \ldots, \mathbf{X}_{n}, \mathbf{Y}_{s}, \ldots, \mathbf{Y}_{m}\right)$ is an arbitrary complex vector function with values in $\mathscr{V}$ and if $k-s \leq 0$, then $h$ is an arbitrary complex vector function only of $\mathbf{X}_{1}, \ldots, \mathbf{X}_{n}$ with values in the same space $\mathscr{V}$.

Proof. We should prove that $f=F(h)$ is a solution of (4.1), where $h$ is an arbitrary complex vector function with values in $\mathscr{V}$.

Indeed, we have

$$
D(f)=D(F(h))=b(h)=0
$$

from where it follows that $E(f)=P(D(f))=\mathbf{O}$, which we were required to prove.

Next, we will solve the functional equation

$$
a_{1} f\left(\mathbf{X}_{1}, \mathbf{X}_{2}, \mathbf{X}_{3}, \mathbf{Y}_{1}, \mathbf{Y}_{2}\right)+a_{2} f\left(\mathbf{X}_{2}, \mathbf{X}_{3}, \mathbf{X}_{1}, \mathbf{Y}_{2}, \mathbf{Y}_{3}\right)+a_{3} f\left(\mathbf{X}_{3}, \mathbf{X}_{1}, \mathbf{X}_{2}, \mathbf{Y}_{3}, \mathbf{Y}_{1}\right)=\mathbf{O} .
$$

By a procedure similar to that in the first section, we may prove the following lemma.

LEMMA 4.3. The functional equation (4.20) is equivalent to the equation

(I) $f\left(\mathbf{X}_{1}, \mathbf{X}_{2}, \mathbf{X}_{3}, \mathbf{Y}_{1}, \mathbf{Y}_{2}\right)=\mathbf{O}$ if $a_{1}+a_{2}+a_{3} \neq 0,\left(a_{1}-a_{2}\right)^{2}+\left(a_{2}-a_{3}\right)^{2}+\left(a_{3}-\right.$ $\left.a_{1}\right)^{2} \neq 0$

(II) $f\left(\mathbf{X}_{1}, \mathbf{X}_{2}, \mathbf{X}_{3}, \mathbf{Y}_{1}, \mathbf{Y}_{2}\right)+f\left(\mathbf{X}_{2}, \mathbf{X}_{3}, \mathbf{X}_{1}, \mathbf{Y}_{2}, \mathbf{Y}_{3}\right)+f\left(\mathbf{X}_{3}, \mathbf{X}_{1}, \mathbf{X}_{2}, \mathbf{Y}_{3}, \mathbf{Y}_{1}\right)=\mathbf{O}$ or $f\left(\mathbf{X}_{1}, \mathbf{X}_{2} \mathbf{X}_{3}, \mathbf{Y}_{1}, \mathbf{Y}_{2}\right)-\omega_{3} f\left(\mathbf{X}_{2}, \mathbf{X}_{3}, \mathbf{X}_{1}, \mathbf{Y}_{2}, \mathbf{Y}_{3}\right)=\mathbf{O}$ if $a_{1}+a_{2}+a_{3} \neq 0$, and $\left(a_{1}-\right.$ $\left.a_{2}\right)^{2}+\left(a_{2}-a_{3}\right)^{2}+\left(a_{3}-a_{1}\right)^{2}=0$;

(III) $f\left(\mathbf{X}_{1}, \mathbf{X}_{2}, \mathbf{X}_{3}, \mathbf{Y}_{1}, \mathbf{Y}_{2}\right)-f\left(\mathbf{X}_{2}, \mathbf{X}_{3}, \mathbf{X}_{1}, \mathbf{Y}_{2}, \mathbf{Y}_{3}\right)=\mathbf{O}$ if $a_{1}+a_{2}+a_{3}=0,\left(a_{1}-a_{2}\right)^{2}+$ $\left(a_{2}-a_{3}\right)^{2}+\left(a_{3}-a_{1}\right)^{2} \neq 0$

(IV) $\mathbf{O}=\mathbf{O}$ or $f\left(\mathbf{X}_{1}, \mathbf{X}_{2}, \mathbf{X}_{3}, \mathbf{Y}_{1}, \mathbf{Y}_{2}\right)+\omega_{3} f\left(\mathbf{X}_{2}, \mathbf{X}_{3}, \mathbf{X}_{1}, \mathbf{Y}_{2}, \mathbf{Y}_{3}\right)+\omega_{3}^{2} f\left(\mathbf{X}_{3}, \mathbf{X}_{1}, \mathbf{X}_{2}, \mathbf{Y}_{3}, \mathbf{Y}_{1}\right)$ $=\mathbf{O}$ if $a_{1}+a_{2}+a_{3}=0$, and $\left(a_{1}-a_{2}\right)^{2}+\left(a_{2}-a_{3}\right)^{2}+\left(a_{3}-a_{1}\right)^{2}=0$. 
Proposition 4.4. The functional equation

$$
f\left(\mathbf{X}_{1}, \mathbf{X}_{2}, \mathbf{X}_{3}, \mathbf{Y}_{1}, \mathbf{Y}_{2}\right)-f\left(\mathbf{X}_{2}, \mathbf{X}_{3}, \mathbf{X}_{1}, \mathbf{Y}_{2}, \mathbf{Y}_{3}\right)=\mathbf{O}
$$

has a general solution

$$
f\left(\mathbf{X}_{1}, \mathbf{X}_{2}, \mathbf{X}_{3}, \mathbf{Y}_{1}, \mathbf{Y}_{2}\right)=h\left(\mathbf{X}_{1}, \mathbf{X}_{2}, \mathbf{X}_{3}\right)+h\left(\mathbf{X}_{2}, \mathbf{X}_{3}, \mathbf{X}_{1}\right)+h\left(\mathbf{X}_{3}, \mathbf{X}_{1}, \mathbf{X}_{2}\right),
$$

where $h$ is an arbitrary complex vector function of the variables $\mathbf{X}_{1}, \mathbf{X}_{2}, \mathbf{X}_{3}$ with values in $\mathcal{V}$.

Proof. The given equation may be written in the following form:

$$
f\left(\mathbf{X}_{1}, \mathbf{X}_{2}, \mathbf{X}_{3}, \mathbf{Y}_{1}, \mathbf{Y}_{2}\right)=f\left(\mathbf{X}_{2}, \mathbf{X}_{3}, \mathbf{X}_{1}, \mathbf{Y}_{2}, \mathbf{Y}_{3}\right) .
$$

The left-hand side of the equation is independent of $\mathbf{Y}_{3}$ and the right-hand side is independent of $\mathbf{Y}_{1}$, so we have

$$
\begin{aligned}
& f\left(\mathbf{X}_{1}, \mathbf{X}_{2}, \mathbf{X}_{3}, \mathbf{Y}_{1}, \mathbf{Y}_{2}\right)=F\left(\mathbf{X}_{1}, \mathbf{X}_{2}, \mathbf{X}_{3}, \mathbf{Y}_{2}\right), \\
& f\left(\mathbf{X}_{2}, \mathbf{X}_{3}, \mathbf{X}_{1}, \mathbf{Y}_{2}, \mathbf{Y}_{3}\right)=F\left(\mathbf{X}_{1}, \mathbf{X}_{2}, \mathbf{X}_{3}, \mathbf{Y}_{2}\right) .
\end{aligned}
$$

On the other hand, from (4.24) we find

$$
f\left(\mathbf{X}_{2}, \mathbf{X}_{3}, \mathbf{X}_{1}, \mathbf{Y}_{2}, \mathbf{Y}_{3}\right)=F\left(\mathbf{X}_{2}, \mathbf{X}_{3}, \mathbf{X}_{1}, \mathbf{Y}_{3}\right),
$$

thus we have

$$
F\left(\mathbf{X}_{1}, \mathbf{X}_{2}, \mathbf{X}_{3}, \mathbf{Y}_{2}\right)=F\left(\mathbf{X}_{2}, \mathbf{X}_{3}, \mathbf{X}_{1}, \mathbf{Y}_{3}\right) .
$$

Since the left-hand side of the above equation is independent of $\mathbf{Y}_{3}$ and the righthand side is independent of $\mathbf{Y}_{2}$, we obtain

$$
F\left(\mathbf{X}_{1}, \mathbf{X}_{2}, \mathbf{X}_{3}, \mathbf{Y}_{2}\right)=G\left(\mathbf{X}_{1}, \mathbf{X}_{2}, \mathbf{X}_{3}\right) .
$$

On the basis of equality (4.28), formula (4.24) becomes

$$
f\left(\mathbf{X}_{1}, \mathbf{X}_{2}, \mathbf{X}_{3}, \mathbf{Y}_{1}, \mathbf{Y}_{2}\right)=G\left(\mathbf{X}_{1}, \mathbf{X}_{2}, \mathbf{X}_{3}\right) .
$$

Formula (4.29) gives a solution of the equation if and only if

$$
G\left(\mathbf{X}_{1}, \mathbf{X}_{2}, \mathbf{X}_{3}\right)-G\left(\mathbf{X}_{2}, \mathbf{X}_{3}, \mathbf{X}_{1}\right)=\mathbf{O},
$$

whose general solution is given by

$$
G\left(\mathbf{X}_{1}, \mathbf{X}_{2}, \mathbf{X}_{3}\right)=h\left(\mathbf{X}_{1}, \mathbf{X}_{2}, \mathbf{X}_{3}\right)+h\left(\mathbf{X}_{2}, \mathbf{X}_{3}, \mathbf{X}_{1}\right)+h\left(\mathbf{X}_{3}, \mathbf{X}_{1}, \mathbf{X}_{2}\right) .
$$

Therefore, the general solution of the equation is

$$
f\left(\mathbf{X}_{1}, \mathbf{X}_{2}, \mathbf{X}_{3}, \mathbf{Y}_{1}, \mathbf{Y}_{2}\right)=h\left(\mathbf{X}_{1}, \mathbf{X}_{2}, \mathbf{X}_{3}\right)+h\left(\mathbf{X}_{2}, \mathbf{X}_{3}, \mathbf{X}_{1}\right)+h\left(\mathbf{X}_{3}, \mathbf{X}_{1}, \mathbf{X}_{2}\right) .
$$


On the basis of the previous results, the following theorem holds.

THEOREM 4.5. The general solution of the equation

$$
a_{1} f\left(\mathbf{X}_{1}, \mathbf{X}_{2}, \mathbf{X}_{3}, \mathbf{Y}_{1}, \mathbf{Y}_{2}\right)+a_{2} f\left(\mathbf{X}_{2}, \mathbf{X}_{3}, \mathbf{X}_{1}, \mathbf{Y}_{2}, \mathbf{Y}_{3}\right)+a_{3} f\left(\mathbf{X}_{3}, \mathbf{X}_{1}, \mathbf{X}_{2}, \mathbf{Y}_{3}, \mathbf{Y}_{1}\right)=\mathbf{O}
$$

is given by the formulas

(1) $f\left(\mathbf{X}_{1}, \mathbf{X}_{2}, \mathbf{X}_{3}, \mathbf{Y}_{1}, \mathbf{Y}_{2}\right) \equiv \mathbf{O}$ if $a_{1}+a_{2}+a_{3} \neq 0$, and $\left(a_{1}-a_{2}\right)^{2}+\left(a_{2}-a_{3}\right)^{2}+\left(a_{3}-\right.$ $\left.a_{1}\right)^{2} \neq 0$

(2) $f\left(\mathbf{X}_{1}, \mathbf{X}_{2}, \mathbf{X}_{3}, \mathbf{Y}_{1}, \mathbf{Y}_{2}\right)=h\left(\mathbf{X}_{1}, \mathbf{X}_{2}, \mathbf{X}_{3}, \mathbf{Y}_{1}\right)-h\left(\mathbf{X}_{2}, \mathbf{X}_{3}, \mathbf{X}_{1}, \mathbf{Y}_{2}\right)$ or $f\left(\mathbf{X}_{1}, \mathbf{X}_{2}, \mathbf{X}_{3}, \mathbf{Y}_{1}, \mathbf{Y}_{2}\right)$ $=h\left(\mathbf{X}_{1}, \mathbf{X}_{2}, \mathbf{X}_{3}\right)+\omega_{3} h\left(\mathbf{X}_{2}, \mathbf{X}_{3}, \mathbf{X}_{1}\right)+\omega_{3}^{2} h\left(\mathbf{X}_{3}, \mathbf{X}_{1}, \mathbf{X}_{2}\right)$ if $a_{1}+a_{2}+a_{3} \neq 0$, and $\left(a_{1}-\right.$ $\left.a_{2}\right)^{2}+\left(a_{2}-a_{3}\right)^{2}+\left(a_{3}-a_{1}\right)^{2}=0$

(3) $f\left(\mathbf{X}_{1}, \mathbf{X}_{2}, \mathbf{X}_{3}, \mathbf{Y}_{1}, \mathbf{Y}_{2}\right)=h\left(\mathbf{X}_{1}, \mathbf{X}_{2}, \mathbf{X}_{3}\right)+h\left(\mathbf{X}_{2}, \mathbf{X}_{3}, \mathbf{X}_{1}\right)+h\left(\mathbf{X}_{3}, \mathbf{X}_{1}, \mathbf{X}_{2}\right)$ if $a_{1}+a_{2}+a_{3}=$ $0,\left(a_{1}-a_{2}\right)^{2}+\left(a_{2}-a_{3}\right)^{2}+\left(a_{3}-a_{1}\right)^{2} \neq 0$;

(4) $f\left(\mathbf{X}_{1}, \mathbf{X}_{2}, \mathbf{X}_{3}, \mathbf{Y}_{1}, \mathbf{Y}_{2}\right)=h\left(\mathbf{X}_{1}, \mathbf{X}_{2}, \mathbf{X}_{3}, \mathbf{Y}_{1}, \mathbf{Y}_{2}\right)$ or $f\left(\mathbf{X}_{1}, \mathbf{X}_{2}, \mathbf{X}_{3}, \mathbf{Y}_{1}, \mathbf{Y}_{2}\right)=h\left(\mathbf{X}_{1}, \mathbf{X}_{2}\right.$, $\left.\mathbf{X}_{3}, \mathbf{Y}_{1}\right)-\omega_{3} h\left(\mathbf{X}_{2}, \mathbf{X}_{3}, \mathbf{X}_{1}, \mathbf{Y}_{2}\right)$ if $a_{1}+a_{2}+a_{3}=0$, and $\left(a_{1}-a_{2}\right)^{2}+\left(a_{2}-a_{3}\right)^{2}+$ $\left(a_{3}-a_{1}\right)^{2}=0$, where $h$ is arbitrary complex vector function with values in $\mathscr{V}$.

ACKNOwledgement. I wish to express sincere thanks to Prof. V. Covachev (Institute of Mathematics, Bulgarian Academy of Sciences). His suggestions and remarks have been of much assistance in the preparation of the paper.

\section{REFERENCES}

[1] I. B. Risteski, Solution of a class of complex vector linear functional equations, Missouri J. Math. Sci. 13 (2001), no. 3, 195-203.

[2] I. B. Risteski and V. Covachev, On some general classes of partial linear complex vector functional equations, SUT J. Math. 36 (2000), no. 2, 105-146.

ICE B. Risteski: 2 Milepost Place \# 606, Toronto M4H 1C7, Canada

E-mail address: iceristeski@hotmai 1 . com 


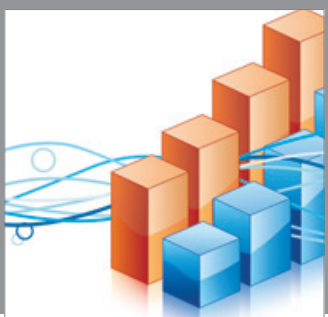

Advances in

Operations Research

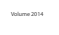

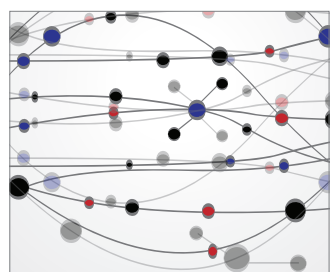

\section{The Scientific} World Journal
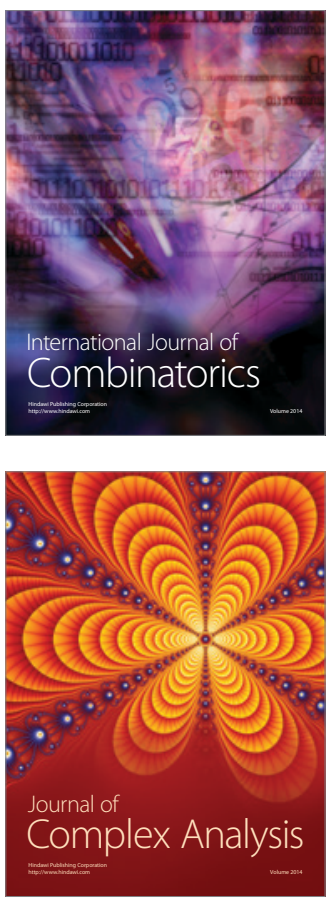

International Journal of

Mathematics and

Mathematical

Sciences
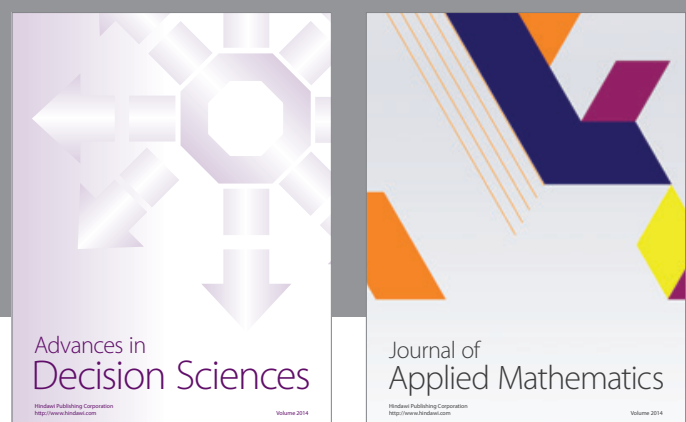

Journal of

Applied Mathematics
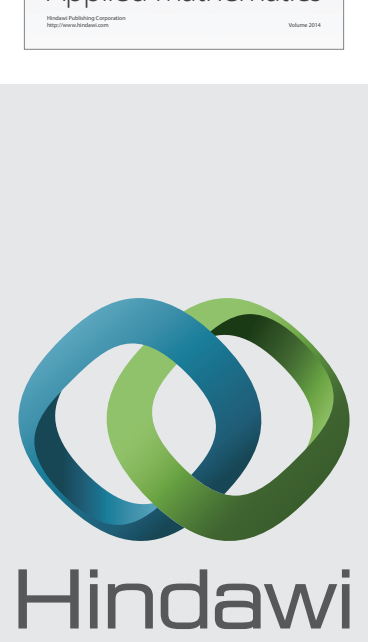

Submit your manuscripts at http://www.hindawi.com
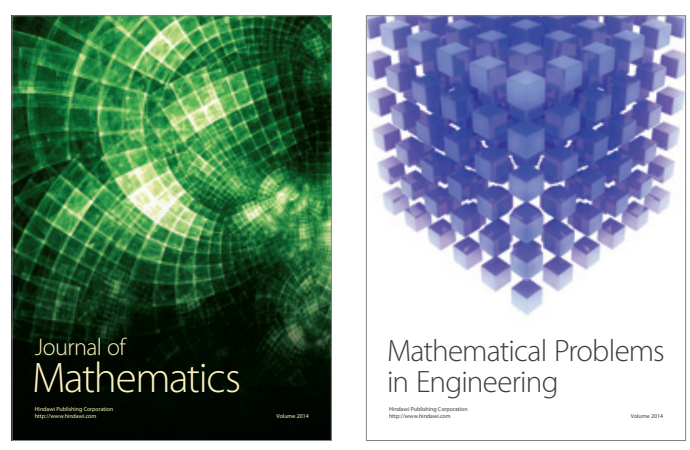

Mathematical Problems in Engineering
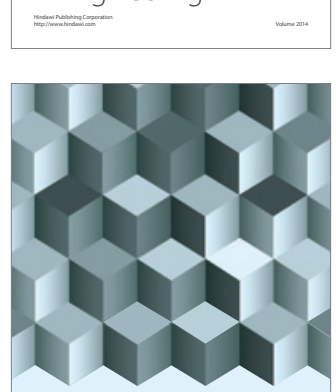

Journal of

Function Spaces
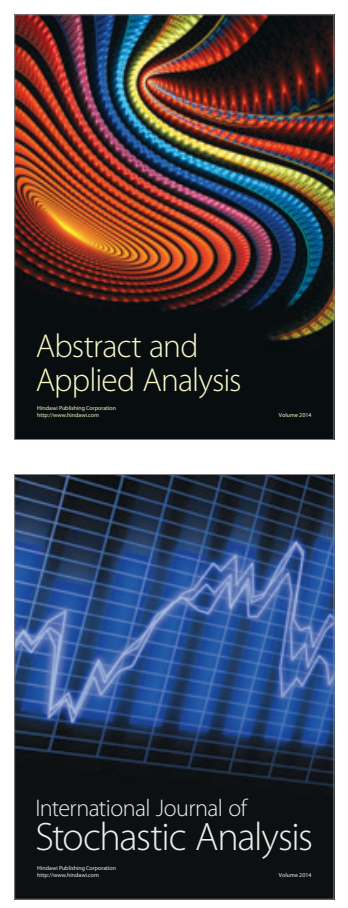

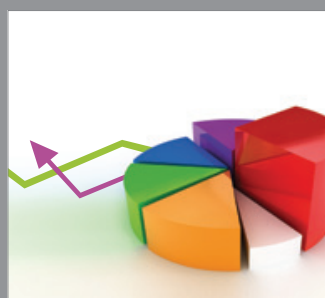

ournal of

Probability and Statistics

Promensencen
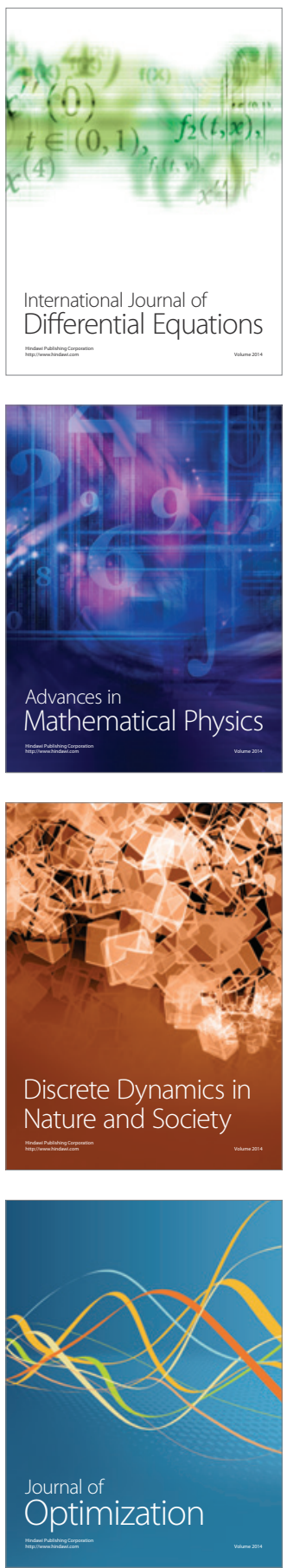